\title{
Construction of gait adaptation model in human splitbelt treadmill walking
}

\author{
Yuji Otoda ${ }^{\mathrm{a} *}$, Hiroshi Kimura ${ }^{\mathrm{b}}$ and Kunikatsu Takase ${ }^{\mathrm{a}}$ \\ ${ }^{a}$ University of Electro-Communications, 1-5-1 Chofugaoka, Chofu, Tokyo 182-8585, Japan; \\ ${ }^{b}$ Kyoto Institute of Technology, Matsugasaki, Sakyo-ku, Kyoto, Japan
}

(Received 14 September 2008; final version received 3 April 2009)

\begin{abstract}
There are a huge number of studies that measure kinematics, dynamics, the oxygen uptake and so on in human walking on the treadmill. Especially in walking on the splitbelt treadmill where the speed of the right and left belt is different, remarkable differences in kinematics are seen between normal and cerebellar disease subjects. In order to construct the gait adaptation model of such human splitbelt treadmill walking, we proposed a simple control model and made a newly developed 2D biped robot walk on the splitbelt treadmill. We combined the conventional limit-cycle based control consisting of joint PD-control, cyclic motion trajectory planning and a stepping reflex with a newly proposed adjustment of P-gain at the hip joint of the stance leg. We showed that the data of robot (normal subject model and cerebellum disease subject model) experiments had high similarities with the data of normal subjects and cerebellum disease subjects experiments carried out by Reisman et al. (2005) and Morton and Bastian (2006) in ratios and patterns. We also showed that P-gain at the hip joint of the stance leg was the control parameter of adaptation for symmetric gaits in splitbelt walking and P-gain adjustment corresponded to muscle stiffness adjustment by the cerebellum. Consequently, we successfully proposed the gait adaptation model in human splitbelt treadmill walking and confirmed the validity of our hypotheses and the proposed model using the biped robot.
\end{abstract}

Keywords: biped robot; model of human walking; splitbelt treadmill; gait adaptation; stepping reflex; P-gain adjustment

\section{Introduction}

Recently, there have been several trials to use robotics as a tool for neuroscience (Schaal et al. 2008), especially in locomotion studies (Ijspeert et al. 2007; Ijspeert 2008; Maufroy et al. 2008). In the case of bipedal legged locomotion, human walking had been investigated intensively over several decades. There are a huge number of studies that measure kinematics, dynamics, the oxygen uptake and so on in human walking on the treadmill (Reisman et al. 2005; Morton and Bastian 2006; Ilg et al. 2007). Especially in walking on the splitbelt treadmill where the speed of the right and left belt is different, remarkable differences in kinematics are seen between normal and cerebellar disease subjects (Morton and Bastian 2006). In order to understand the mechanisms behind such phenomena, it is useful to construct the control model of human walking, simulate it using a musculoskeletal model and compare the simulation results with the results of human experiments. But since it is difficult to simulate friction, collision with ground, effects of elastic materials and so on, we would like to carry out experiments using a real machine (robot) rather than computer simulations. We use a newly developed 2D biped robot called 'Tetsuro' to construct the control model of human walking and investigate how it works.

In this article, firstly we introduce the results of human (normal and cerebellar disease subjects) splitbelt walking
(Reisman et al. 2005; Morton and Bastian 2006). Secondly we describe the conventional limit-cycle based control consisting of joint PD-control, cyclic motion trajectory planning based on the inverted pendulum, and a stepping reflex for forward speed and posture control, while modeling motion generation and control at the spinal cord and the brainstem. Thirdly, in order to enable Tetsuro to walk on the splitbelt treadmill autonomously, we propose P-gain adjustment at the hip joint of the stance leg, while modeling motion adjustment at the cerebellum. Finally, we compare the results of Tetsuro experiments with those of human (normal and cerebellar disease subjects) experiments (Reisman et al. 2005; Morton and Bastian 2006), and discuss the validity of the proposed control model. High similarities in ratios and patterns of the measured indexes imply the validity of our hypotheses and the proposed model.

\section{Human splitbelt treadmill walking and hypotheses}

In this section, we introduce the results of human (normal and cerebellar disease subjects) splitbelt walking by Reisman et al. (2005) and Morton and Bastian (2006) and their hypotheses.

\subsection{Definitions}

A human walks on the treadmill equipped with two belts moving either at same speed ('tied'configuration) or at

\footnotetext{
*Corresponding author. Email: yujioto@kimura.is.uec.ac.jp
} 


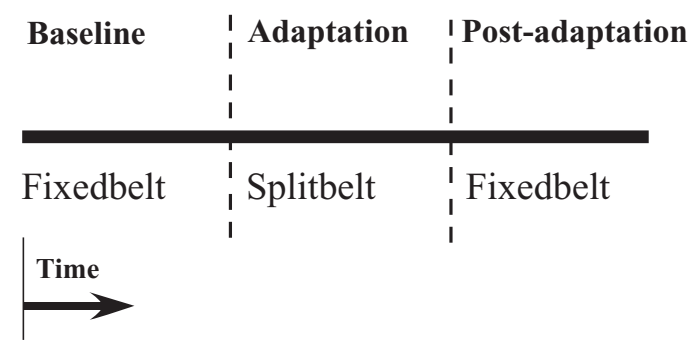

Figure 1. Three stages in experiments of splitbelt treadmill.

different speed ('splitbelt'configuration). The speed of a treadmill belt is either 'slow' $(0.5 \mathrm{~m} / \mathrm{s})$ or 'fast' $(1.0 \mathrm{~m} / \mathrm{s})$. In the tied configuration, both treadmill belts are at slow speed. In the splitbelt configuration, one treadmill belt is set at slow speed and the other is set at fast speed. The time course of the experiment (Figure 1) is divided into three stages. In the 'baseline' stage, the treadmill is in the tied configuration. In the 'adaptation' stage, the treadmill is in the splitbelt configuration. In the 'post-adaptation' stage, the treadmill is in the tied configuration again.

There are four kinematic variables measured during treadmill walking. The definitions of the stride length and the step length are shown in Figure 2. The duty ratio of a leg is defined as the percentage of the stance period of the leg within the walking cyclic period. The ratio of the double legs stance period (RDLSP) is defined as the percentage of the DLSP within the walking cyclic period. All of these variables are measured for both legs.

The legs on slow and fast moving belts in the splitbelt configuration are named as 'slow' and 'fast' legs, respectively. Although both legs are moving at the slow speed in the baseline and post-adaptation stages, they keep these names. The DLSP occurring at the end of the slow leg stance phase and beginning at the contact of the fast leg is named as the 'slow' DLSP. The DLSP occurring at the end of the fast leg stance phase and beginning at the contact of the slow leg is named as the 'fast' DLSP. The stride length and the duty ratio are measured from a single leg and called 'intralimb' indexes. The step length difference and the RDLSP difference are calculated by 'fast' minus 'slow' (subtracting the value of 'slow' from the value of 'fast'). Those are measured depending on both legs and called 'interlimb' indexes.

\subsection{Normal subjects splitbelt treadmill walking}

The stride length and the duty ratio measured in normal subjects' experiments are shown in Figure 3, and the step length difference and the RDLSP difference in normal subjects' experiments are shown in Figure 4 (modified from Morton and Bastian (2006)). Each circle in those figures means the value of the index measured at single walking cycle.
Let us see the values of the stride length and the duty ratio in Figure 3. In the adaptation stage, the stride length became much larger in the fast leg and a little smaller in the slow leg, and the duty ratio became lower in the fast leg and almost constant in the slow leg. Let us see the patterns of those indexes in Figure 3. Those indexes were almost constant in the baseline stage, quickly changed at the beginning of the adaptation stage and were kept almost constant within the adaptation stage, and quickly returned almost to the original values at the beginning of the postadaptation stage.

Let us see the values and the patterns of the step length difference and the RDLSP difference in Figure 4. In the baseline stage, we can see that the step length difference was almost constant, and that the RDLSP difference was not constant ${ }^{1}$. We can see that those indexes quickly changed ${ }^{2}$ at the beginning of the adaptation stage and gradually returned almost to the original values in the late adaptation stage. We can see that those indexes quickly changed ${ }^{3}$ at the beginning of the post-adaptation stage and gradually returned almost to the original values in the late post-adaptation stage. Here are two interesting phenomena. One is that those indexes gradually returned almost to the values near zero in the late adaptation stage in spite of difference in the speed of two belts. This meant that normal subjects preferred a symmetric gait even in the splitbelt configuration. Another is that those indexes quickly changed at the beginning of the post-adaptation stage even though the speed of two belts became same. This is one of well-known phenomena called 'aftereffects'.

\subsection{Cerebellar disease subjects splitbelt treadmill walking}

The stride length and the duty ratio measured in cerebellar disease subjects experiments are shown in Figure 5, and the step length difference and the RDLSP difference in cerebellar disease subjects experiments are shown in Figure 6 (modified from Morton and Bastian (2006)).

In Figure 5(a), at the beginning of the adaptation stage, cerebellar disease subjects made quick reactive adjustments of the stride length of both legs so that the stride length increased substantially on the fast leg and decreased slightly on the slow leg as observed in normal subjects (Figure 3(a)). At the beginning of the post-adaptation stage, cerebellar disease subjects again made quick reactive adjustments of the stride length of both legs while returning almost to baseline levels as observed in normal subjects

\footnotetext{
${ }^{1}$ We have not yet understood the reason.

${ }^{2}$ The value of the slow leg became much larger. This meant an asymmetric gait.

${ }^{3}$ The value of the fast leg became much larger. This meant another asymmetric gait.
} 


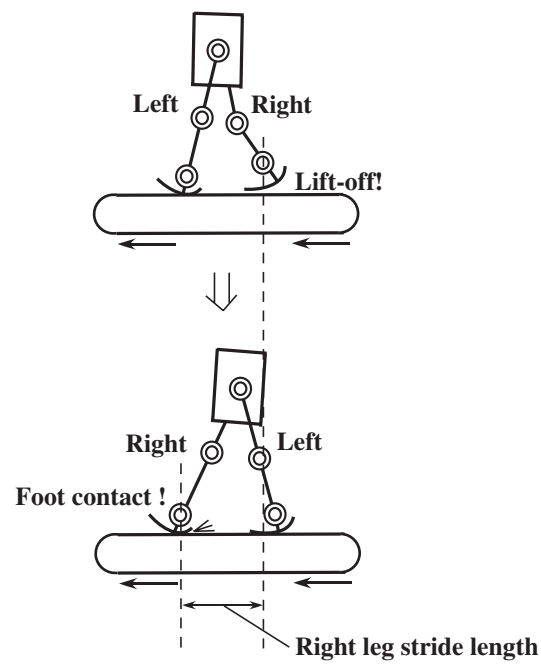

(a) Definition of stride length

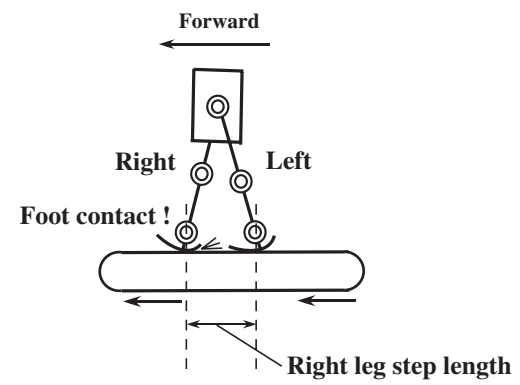

(b) Definition of step length

Figure 2. Definitions of the stride length (left) and the step length (right). The stride length is defined as the distance travelled by the ankle joint of one leg from the instant of lift-off to the instant of foot contact of the leg. The step length is defined as the distance between positions of the ankle joints of swing and stance legs at the instant of foot contact of the swing leg.

(Figure 3(a)). In Figure 5(b), cerebellar disease subjects showed more stride-to-stride variability in the values of the duty ratio, but were still clearly able to generate the corrective response quickly as being observed in normal subjects (Figure 3(b)).

While comparing the step length difference in cerebellar disease subjects (Figure 6(a)) with that in normal subjects (Figure 4(a)), we can see that it quickly changed at the be- ginning of the adaptation stage in a similar way. But we can see neither gradually returning to the original values in the late adaptation stage nor aftereffect at the beginning of the post-adaptation stage in cerebellar disease subjects. While comparing the RDLSP difference in cerebellar disease subjects (Figure 6(b)) with that in normal subjects (Figure 4(b)), we can see neither adjustment nor aftereffect in cerebellar disease subjects. a

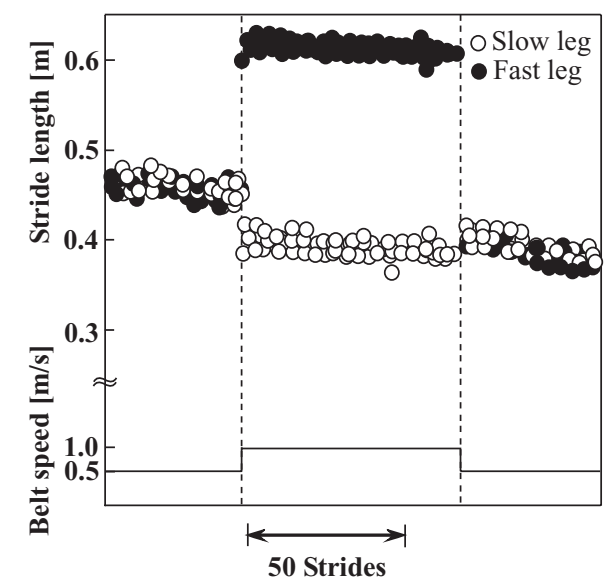

b

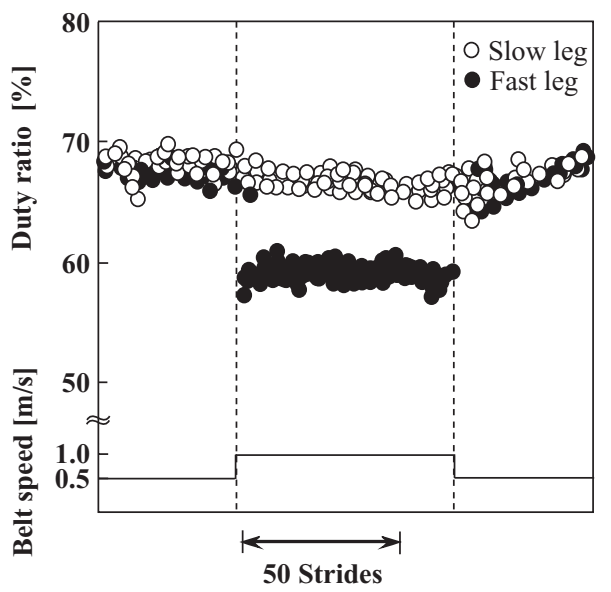

Figure 3. Results of human (normal subject) experiment (modified from Morton and Bastian (2006)). The stride length (A) and the duty ratio (B) in splitbelt treadmill walking are shown, where speed of belts were $0.5 \mathrm{~m} / \mathrm{s}$ at both left and right belts in the baseline stage, 0.5 $\mathrm{m} / \mathrm{s}$ at the left belt and $1.0 \mathrm{~m} / \mathrm{s}$ at the right belt in the adaptation stage, and $0.5 \mathrm{~m} / \mathrm{s}$ at both left and right belts in the post-adaptation stage. Speed of the fast belt is also shown. 


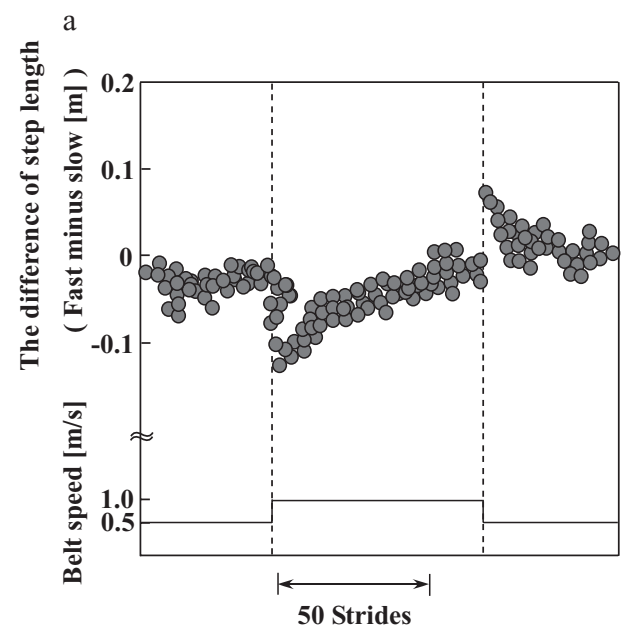

b

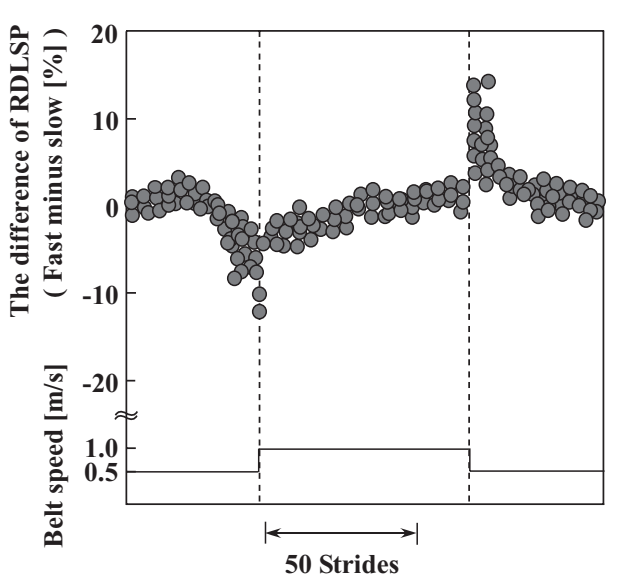

Figure 4. Results of human (normal subject) experiment (modified from Morton and Bastian (2006)). The step length difference (A) and RDLSP (ratio of the double legs stance period) difference (B) in splitbelt treadmill walking are shown. The time course of belts speed is described in the caption of Figure 3.

\subsection{Hypotheses and control model}

As a conclusion of normal subjects' splitbelt walking experiments Reisman et al. (2005), mentioned that there are two types of neural control independent of each other. One is for intra-limb coordination adjusting the stride length and the duty ratio in the sense that those indexes are calculated from an individual leg. This changes quickly to accommodate splitbelts and shows no aftereffect. Another is for inter-limb coordination adjusting the step length difference and the RDLSP difference in the sense that those indexes change slowly during adaptation and show aftereffects during post-adaptation. They mentioned that such inter-limb coordination might adapt to optimise the relative movement between legs. In addition, they mentioned two possibilities about central neural system regions concerned with such inter-limb coordination. One possibility is spinal networks. Another possibility is cerebellum or brainstem neurons of the vestibulospinal pathways under the control of the cerebellum.

As a conclusion of cerebellar disease subjects' splitbelt walking experiments Morton and Bastian (2006), mentioned that there are two types of adaptation. One is the reactive feedback-driven adaptation which is not impaired by cerebellar damage and predominantly controlled by lower neural centers such as the spinal cord or brainstem. Another is the predictive feedforward adaptation which is significantly disrupted by cerebellar damage. a

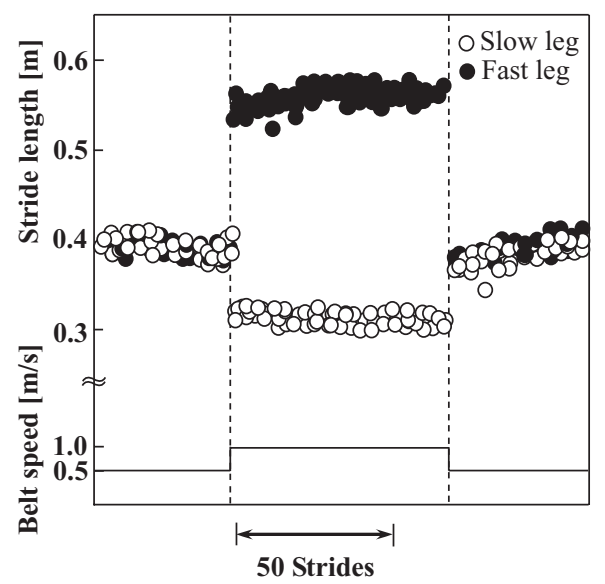

b

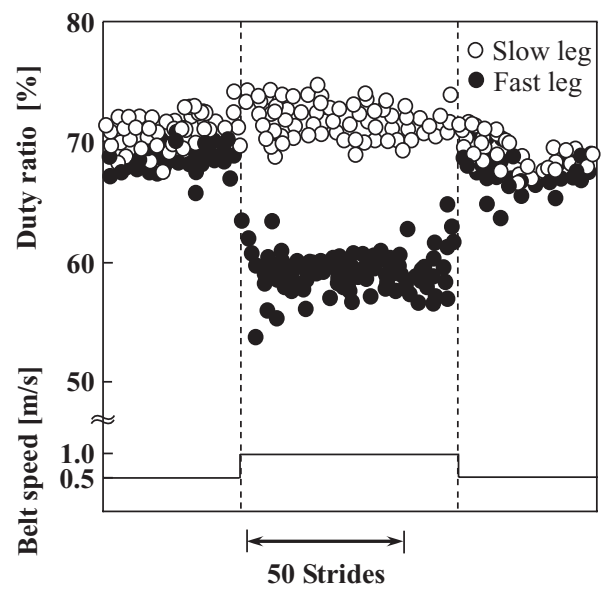

Figure 5. Results of human (cerebellar disease subject) experiment (modified from Morton and Bastian (2006)). The stride length (A) and the duty ratio (B) in splitbelt treadmill walking are shown. The time course of belts speed is described in the caption of Figure 3 . 


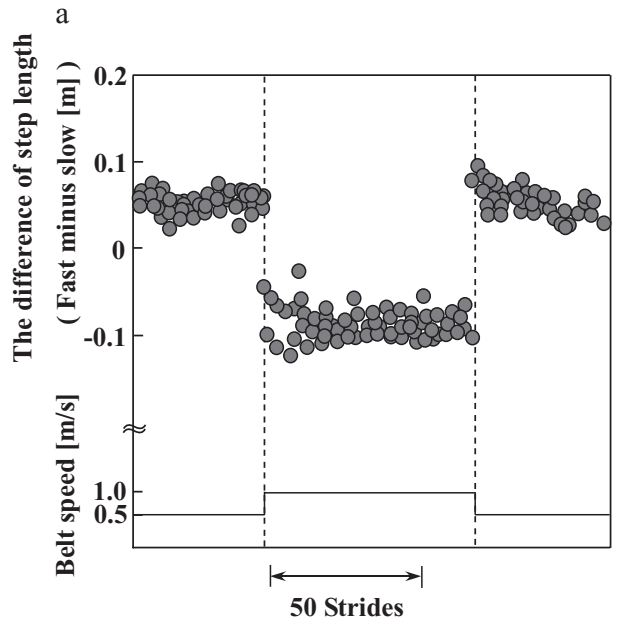

b

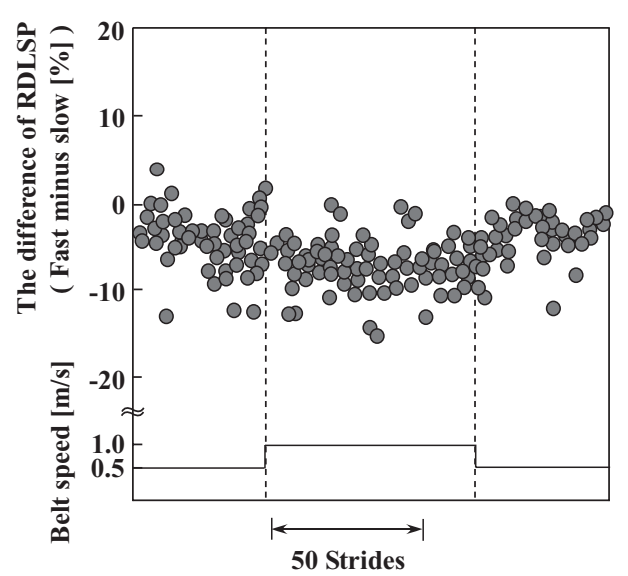

Figure 6. Results of human (cerebellar disease subject) experiment (modified from Morton and Bastian (2006)). The step length difference (A) and RDLSP difference (B) in splitbelt treadmill walking are shown. The time course of belts speed is described in the caption of Figure 3.

As a result of decerebrate cat experiments, it is generally accepted that rhythmic motion of limbs in walking is predominantly generated and controlled by central pattern generators (CPGs) in the spinal cord and reflexes in the spinal cord and brainstem (Orlovsky et al. 1999; Rossignol et al. 2006). We take a hypothesis such that similar neural control is working in human bipedal walking (Ilg et al. 2007; Orlovsky et al. 1999; Rossignol et al. 2006). While referring to such knowledge in physiology and hypotheses by the above mentioned authors we propose the control model for biped robot splitbelt walking (Figure 7) employing the following mechanisms:

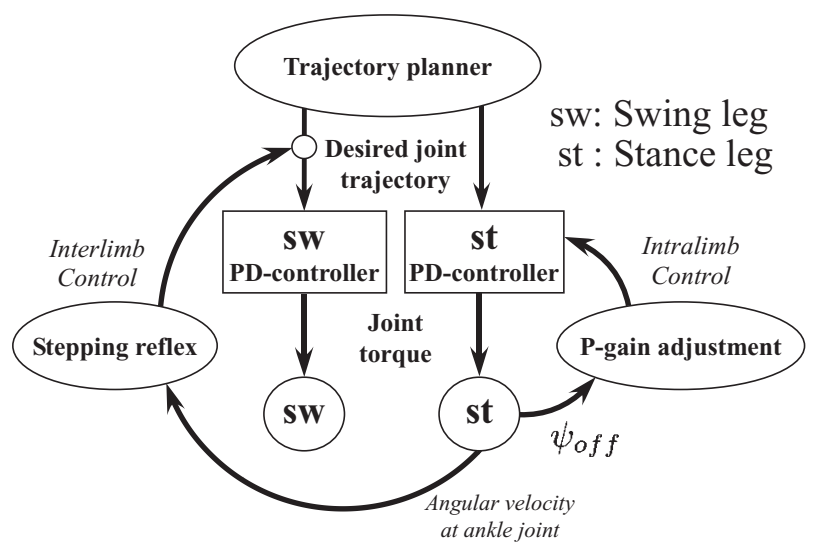

Figure 7. Control diagram of biped robot splitbelt walking. The stepping reflex acts as inter-limb control since hip joint angle of the swing leg is adjusted by ankle joint angular velocity of the stance leg as described in Section 3.2.5. P-gain adjustment acts as intra-limb control since P-gain at hip joint of the stance leg in the next stance phase is adjusted by hip joint angle of the leg at last lift-off as described in Section 3.3.
(1) The cyclic motion trajectory planner as a simple model of CPG for generation of rhythmic motion. ${ }^{4}$

(2) The joint PD controller as a simple model of the tonic stretch reflex in the spinal cord.

(3) The stepping reflex for stabilisation of forward speed and posture as the vestibulospinal reflex ${ }^{5}$ at the brainstem, of which style is inter-limb control as described in Section 3.2.5.

(4) The adjustment of P-gain at hip joint of the stance leg as a simple model of adaptation by the cerebellum, of which style is intra-limb control as described in Section 3.3.

Especially, we newly propose the P-gain adjustment based on knowledge that the cerebellum adjusts the muscle stiffness in a phasic manner (Kandel et al. 1996). The details of each mechanism are described in Section 3 and Appendix A.

\section{Robot splitbelt treadmill walking}

\subsection{Conventional bipedal walking control methods}

In the 80 s and mid-90s, biped robots were actively studied (Miura and Shimoyama 1984; Takanishi et al. 1985; Sano and Furusho 1990; Kajita and Tani 1997). After that, several humanoid robots were developed (Yamaguchi

\footnotetext{
${ }^{4}$ The ability for mutual entrainments (Orlovsky et al. 1999; Rossignol et al. 2006) would be employed in future.

${ }^{5}$ We did not use the output of the rate gyro sensor as vestibule but the ankle joint angular velocity for the stepping reflex, since the output of the rate gyro is a little noisy and the joint angular velocity is more reliable.
} 
and Takanishi 1997; Hirai et al. 1998; Yokoi et al. 2004; Nishiwaki and Kagami 2008), and the interest in biped robots is still growing. Most of those recent humanoid robots have used the Zero Moment Point (ZMP) based control method. Those humanoid robots can walk on level ground and move up- and down-stairs. In this method, since the whole body is accelerated and decelerated by actuators in every step, the motion is not energetically efficient.

On the other hand, utilising mechanical features of the machine and gravity can generate efficient walking, i.e. walking with less output of actuators as far as possible. Such motion is called 'walking using natural dynamics'. A typical example of walking using natural dynamics is passive dynamic-walking down a slope (Collins et al. 2001). A well-designed biped robot has its own natural dynamics that are suitable for efficient walking. Such natural dynamics can be utilised by the limit-cycle based control method. Asano et al. and Sugimoto et al. proposed Energy Referenced Control (Asano et al. 2004) and Delayed Feedback Control (Sugimoto and Osuka 2002), respectively. Wisse and van Frankenhuyzen (2006), Takuma and Hosoda (2006) and Tsujita et al. (2007) achieved efficient walking referring to passive dynamic walking in the pitch plane using pneumatic artificial muscle actuators. Especially (Takuma and Hosoda 2006) and Tsujita et al. (2007), realised the change of walking speed while changing the stiffness at joints by adjusting the on/off timing of valves. Chemori and Alamir (2004) analysed the stability of the achieved walking using the Poincaré section method. There are a lot of studies (Taga et al. 1991; Tomita and Yano 2003; Aoi and Tsuchiya 2005; Morimoto et al. 2008; Endo et al. 2004; Geng et al. 2006) using rhythm generators and their functions of entrainment with the body in order to generate efficient and adaptive walking while constructing the limit-cycle.

Humans can use both the ZMP based control method and the limit-cycle based control method. But it is said that humans use the limit-cycle based control method in order to utilise natural dynamics in normal walking. Therefore, we use the limit-cycle based control method for construction of human splitbelt treadmill walking.

\subsection{Biped robot and tied treadmill walking}

We developed a 2D biped robot called 'Tetsuro' and implemented a simple and conventional limit-cycle based control method consisting of joint PD control, cyclic motion trajectory planning and stepping reflex for tied treadmill walking under disturbance. Although there is nothing novel in the mechanical design of Tetsuro and its control method, we describe details to some extent in this section in order to explain the experimental setup and notations used in the following sections.

\subsubsection{Biped robot: 'Tetsuro'}

Figure 8(a) shows the whole body of Tetsuro. The length of the leg while standing is approximately $40 \mathrm{~cm}$, and the weight of the whole body is $2.3 \mathrm{~kg}$. The lengths of the body, the upper link (thigh) and the lower link (shank) are $6 \mathrm{~cm}$, $18 \mathrm{~cm}$ and $20 \mathrm{~cm}$, respectively. The weights of the body, the upper link and the lower link are $0.7 \mathrm{~kg}, 0.3 \mathrm{~kg}$ and $0.5 \mathrm{~kg}$, respectively. In Figure 8(b), each leg has three DOF (hip, knee and ankle joints around the pitch axis). The rolling motion of the body of Tetsuro is constrained by two parallel pipes with linear bushings (see the snapshots of Tetsuro walking in Appendix A.3). Since friction at contacting points between the constrained plates of the body (Figure 8(a)) and the two linear bushings of the pipes is small, the body has three DOF in the pitch plane and can move smoothly. Therefore, Tetsuro can easily fall down by stumbling of the swing $\operatorname{leg}^{6}$ and large disturbances at worst. The center of gravity of the body is slightly below the hip joint.

The hip, knee and ankle joints are actuated by DC motors of 20,20 and $19 \mathrm{~W}$ via spur gears of which gear ratios are 25,25 and 8.7 , respectively. Since those gear ratios are small, friction at each joint is small and each joint has high backdrivability, Tetsuro can walk efficiently utilising its natural dynamics as described in Appendix A and (Otoda et al. 2007).

A photo encoder is equipped at each joint to measure its angle. A rate gyro sensor is equipped at the body to measure the body pitch angle. Two mechanical switches are equipped as contact sensors beneath the sole of each leg (Figure $8(d)$ ). The cylindroid shape of the sole makes the center of gravity of Tetsuro move forward smoothly in the single leg stance phase.

\subsubsection{PD control}

Output torque at each joint is calculated using simple PD control$^{7}$ expressed by the following equation.

$$
\tau_{j}^{p h}=-k_{j_{-} p}^{p h}\left(\theta_{j_{-} c}^{p h}-\theta_{j_{-} d}^{p h}\right)-k_{j_{-} v}^{p h}\left(\dot{\theta}_{j_{-} c}^{p h}-\dot{\theta}_{j_{-} d}^{p h}\right)
$$

$\tau_{j}^{p h}, \theta_{j_{-c}}^{p h}$ and $\theta_{j_{-}}^{p h}$ are output torque, measured joint angle and desired joint angle at a joint $j \in\{h, k, a\}$ in a phase $p h \in\{s w, s t\}$, respectively, where $h, k$ and $a$ mean hip, knee and angle joint, and $s w$ and $s t$ mean swing and stance phase, respectively (Figure 9). $\dot{\theta}_{j \_c}^{p h}$ and $\dot{\theta}_{j-d}^{p h}$ are measured and desired joint angular velocity. $k_{j_{-} p}^{p h}$ and $k_{j_{-} v}^{p h}$ are $\mathrm{P}$ and $\mathrm{D}$ feedback gain, respectively. In this study, $\dot{\theta}_{j-d}^{p h}$ was set to zero.

\footnotetext{
${ }^{6}$ If a flexor reflex is employed, Tetsuro can avoid falling down by stumbling of the swing leg.

${ }^{7}$ The knee joint of the swing leg in the single leg stance phase is free and this PD control is not used.
} 


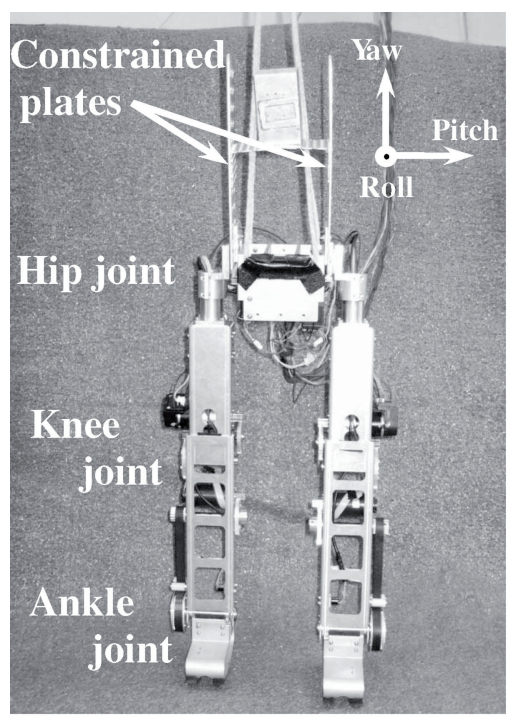

(a)

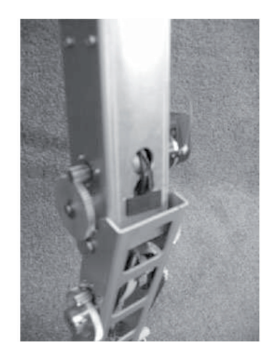

(c)

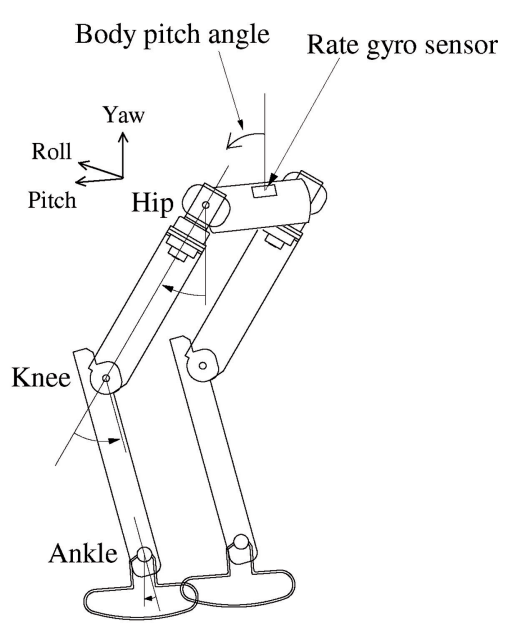

(b)

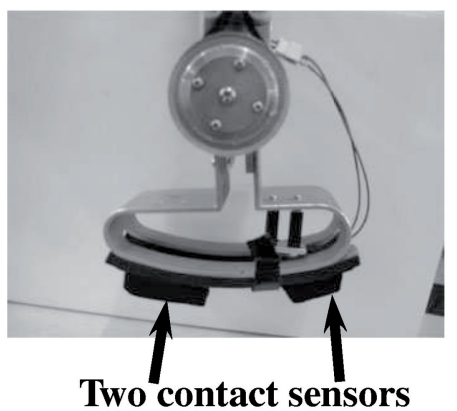

(d)

Figure 8. (a) Photo of Tetsuro, (b) mechanical draft of Tetsuro, (c) photo of knee joint and mechanical stopper, and (d) photo of ankle joint, foot and contact sensors beneath the sole.

\subsubsection{Treadmill}

The treadmill has two belts equipped with a DC motor for each, so that the speed of each belt can be controlled independently. We can change the belt speed from $0 \mathrm{~m} / \mathrm{s}$

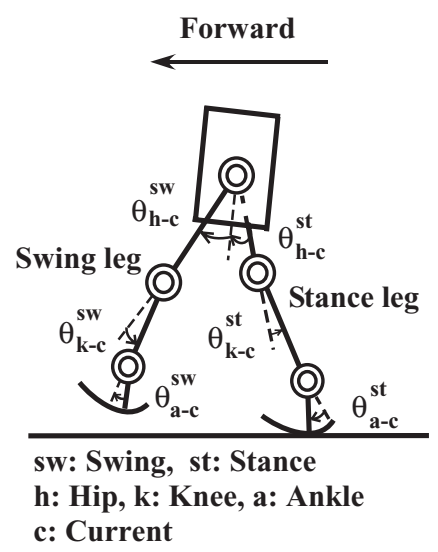

to $0.6 \mathrm{~m} / \mathrm{s}$ in every $0.005 \mathrm{~m} / \mathrm{s}$ step manually. Treadmill acceleration and deceleration rate in switching to the next stage is $1.0 \mathrm{~m} / \mathrm{s}^{2}$.

\subsubsection{Walking on the tied treadmill}

The details of the cyclic motion trajectory planning based on the inverted pendulum, the desired trajectory of each joint and experimental results of tied treadmill walking are described in Appendix A. The values of parameters used in experiments are shown in Appendix B.

\subsubsection{Stepping reflex for speed and posture control}

As an inter-limb reactive control against disturbances, we employ a stepping reflex illustrated in Figure 10, where eq. (2) is used in place of the trajectory of the hip joint of the swing leg in the stance-swing state (Table 1).

$$
\theta_{h_{-} d}^{s w}=\phi(t)+k_{s r} \times\left(\dot{\theta}_{a_{-} c}^{s t}-\dot{\theta}_{a_{-} d}^{s t}\right),
$$




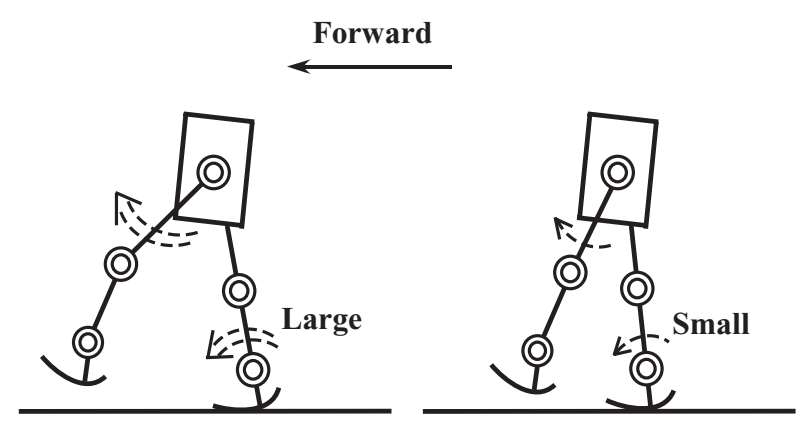

Figure 10. Stepping reflex. The robot changes the touchdown angle of the swing leg according to the ankle joint angular velocity of the stance leg.

where $\phi(t)$ is the trajectory based on the inverted pendulum model (Appendix A.1) and $k_{s r}$ is the gain of the stepping reflex. The desired hip joint angle considering the stepping reflex is decided by the angular velocity of the ankle joint on the vertical axis in the ankle joint so as not to oscillate the desired angle.

Using this stepping reflex, a robot can adjust the touchdown angle of the swing leg according to the change of angular momentum of the whole body. The angular velocity of the stance leg ankle joint itself does not indicate the angular momentum of the whole body. We have coded a filter for the error in the measured angular velocity and used the value through the filter. Since this touchdown angle becomes the initial angle of the stance leg in the next step, the robot can adjust the motion of the stance leg moving as an inverted pendulum approximately while utilising gravity. Moment of inertia of the swing leg around the hip joint is relatively smaller than that of the whole body around the ankle joint of the stance leg. Therefore, the stepping reflex is less energy consumptive than torque adjustment at the ankle joint of the stance leg, which is used for posture control based on ZMP. Consequently, the stepping reflex enables a robot to keep forward speed and posture efficiently against disturbances such as pushing on its back.

Miura and Shimoyama (1984) first confirmed the effectiveness of the stepping reflex using a stilt-type biped robot. They also showed computationally that there exist lower and upper bounds of the stepping reflex gain for stable walking. The value of $k_{s}$ in experiments described in this paper was $0.35^{8}$, which was determined manually. The touchdown angle adjustment such as the stepping reflex is also known as the Raibert's neutral point control (Raibert 1986). The importance of such stepping reflex in human balance control is also reported (Rogers et al. 2003).

We carried out experiments to confirm the effectiveness of the stepping reflex under disturbance. For giving dis-

\footnotetext{
${ }^{8} \mathrm{An}$ inappropriate value of $k_{s r}$ (for example, 0.28) made Tetsuro fall down easily under disturbances.
}

Table 1. Desired trajectory of each joint in the stanceswing state.

\begin{tabular}{ll}
\hline Desired angles & \multicolumn{1}{c}{ Desired trajectories [rad] } \\
\hline$\theta_{h d}^{s w}$ & $\phi(t)$ \\
$\theta_{k-d}^{s w}$ & No control (free) \\
$\theta_{a_{d}}^{s w}$ & $0.2 \quad$ (toe raise) \\
$\theta_{h d}^{s t}$ & $-\phi\left(T_{0}\right)$ (moving the body forward) \\
$\theta_{k-d}^{s s t}$ & -0.07 (lock) \\
$\theta_{a_{-} d}^{s t}$ & $\begin{cases}\phi(t) & \text { (in transitional walking) } \\
0 & \text { (in steady walking) }\end{cases}$ \\
\hline
\end{tabular}

turbance, we prepared a pendulum consisting of the mass $(0.7 \mathrm{Kg}$ in weight) and the string ( $1.4 \mathrm{~m}$ in length) and hanging from the ceiling. We hit Tetsuro on its back with the inelastic collision with the mass after releasing the pendulum at $30^{\circ}$ to the vertical axis in position. Figure 11 shows the experimental result. Each circle in this figure means the touchdown right hip angle of the swing leg measured at single walking cycle. We can see that Tetsuro was pushed forward in the left leg stance phase and the touchdown angle of the right leg was increased according to the increased ankle joint angular velocity of the left leg as the result of the stepping reflex (Figure 11(a)). Consequently, Tetsuro kept forward speed and posture against such disturbance.

\subsection{P-gain adjustment for gait adaptations}

As an intra-limb reactive control for splitbelt walking, we employ adjustment of P-gain at hip joint of the stance leg. Since this P-gain adjustment is implemented on each leg independently, we count the number of steps on each leg separately and the following eq. (3) is applied to each leg independently.

In Figure 12, $\psi_{\text {off }}$ is the hip relative joint angle of the leg at lift-off. Muscle spindles, Golgi tendon organs and the cutaneous receptor on the soles are used to measure hip angle at lift-off in human case. Muscle spindles are sensory receptors within the belly of a muscle, which primarily detect changes in the length of this muscle. They convey length information to the central nervous system via sensory neurons. The Golgi tendon organ is a proprioceptive sensory receptor organ that is located at the insertion of skeletal muscle fibres into the tendons of skeletal muscle. It can measure the tension that each muscle contraction builds up. The P-gain of the hip joint of the leg in the next stance phase as the $n t h$ step is updated in the adaptation and post-adaptation stages using the following equation.

$$
k_{h_{-} p}^{s t}[n]=k_{a g} \times\left(\bar{\psi}_{o f f}-\psi_{o f f}[n-1]\right)+k_{h_{-} p}^{s t}[n-1],
$$

where $k_{a g}$ is the gain for P-gain adjustment, $\bar{\psi}_{o f f}$ is the average of $\psi_{o f f}$ of the leg in the baseline stage and $k_{h_{-}}^{s t}[n]$ is the hip joint p-gain of the leg in the stance phase within 


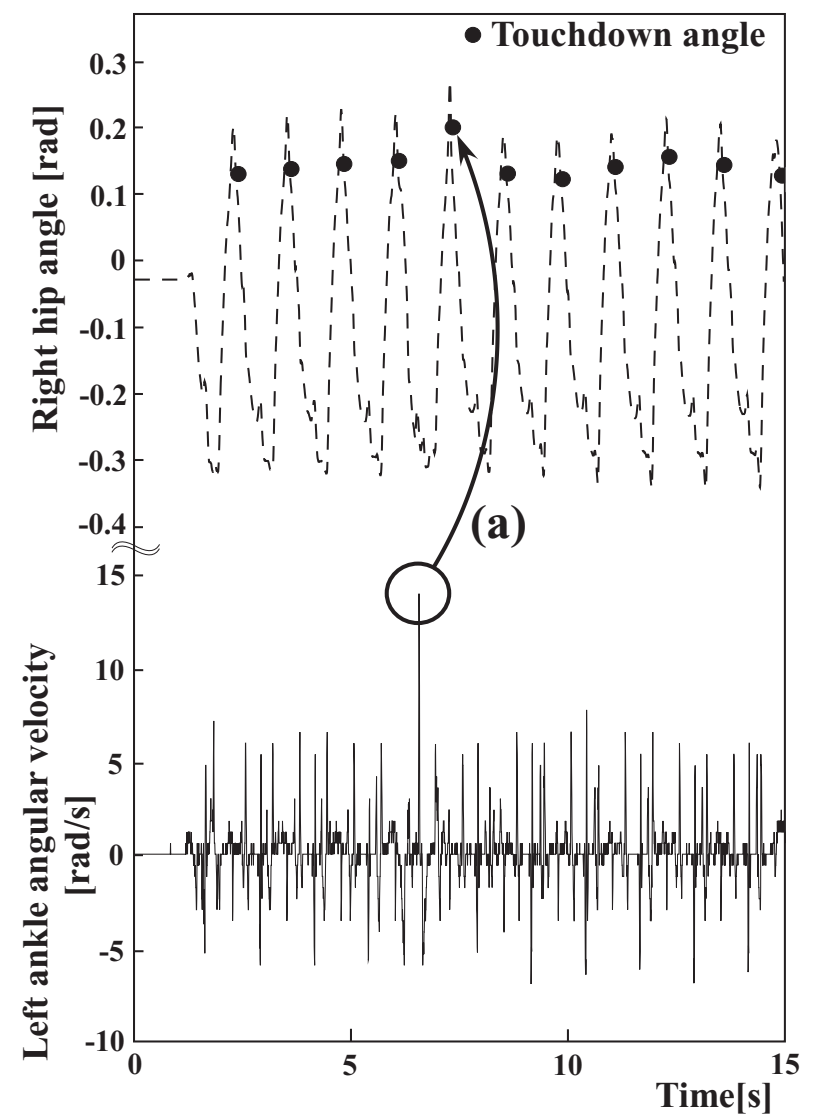

Figure 11. Experimental result in tied treadmill walking with the belt speed: $0.20 \mathrm{~m} / \mathrm{s}$ to see the effectiveness of a stepping reflex against disturbance.

the $n t h$ step. The cerebellum can memorise past gain to adjust the muscle stiffness and average measured hip angle approximately using Muscle spindles, Golgi tendon organs and the cutaneous receptor on the soles in human neural structure (Kandel et al. 1996). We newly propose this mechanism based on the knowledge that the cerebellum adjusts the muscle stiffness and the ongoing movement in a phasic manner.

The reason why such P-gain adjustment enables a robot to walk on the splitbelt treadmill is illustrated in Figure 13.

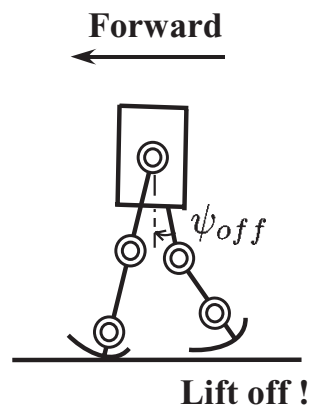

Figure 12. Definition of $\psi_{o f f}$.

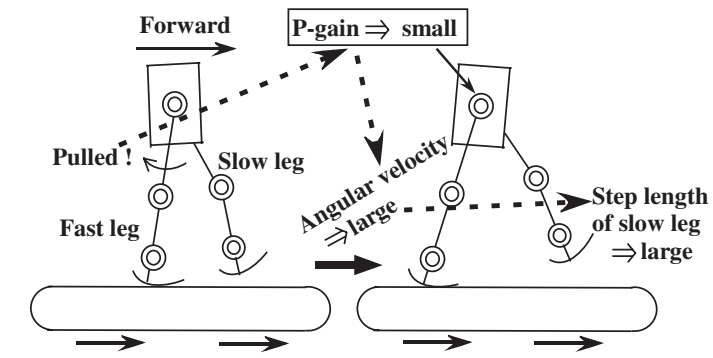

(a) Stance phase of the fast leg

(b) Next stance phase of the fast leg

Figure 13. P-gain adjustment for splitbelt treadmill walking.

When the speed of one belt becomes high in the adaptation period, the fast leg is pulled further backward by the belt in the stance phase (Figure 13(a)) and $\left|\psi_{\text {off }}\right|$ becomes larger. In the next stance phase of the fast leg, as a result of the P-gain adjustment expressed by eq. (3), the stiffness of the hip joint at the fast leg becomes lower, and the fast leg is pulled much further backward (Figure 13(b)). In addition, since the ankle joint angular velocity of the fast leg in the stance phase becomes larger, the touchdown angle of the swing leg becomes larger by the stepping reflex expressed by eq. (2). As a result, the position of the body in the world coordinate is mostly kept constant, and the robot can keep walking on the splitbelt treadmill.

\section{Experiments of splitbelt treadmill walking and discussions}

The time course of robot experiments was same with the one shown in Figure 1. The speed of the treadmill belt was either 'slow' $(0.15 \mathrm{~m} / \mathrm{s})$ or 'fast' $(0.30 \mathrm{~m} / \mathrm{s})$. The period of each stage was $15 \mathrm{~s}$.

\subsection{Normal subject model}

We made Tetsuro walk on the splitbelt treadmill in the above described experimental setup with P-gain adjustment described in Section 3.3. Tetsuro calculated $\bar{\psi}_{\text {off }}$ in the baseline stage, autonomously detected the switch from the baseline stage to the adaptation stage by detecting the change of lift-off timing of legs caused by the change of belt speed and started P-gain adjustment expressed by eq. (3). Tetsuro did not change its control method at the switch from the adaptation stage to the post-adaptation stage.

The stride length and the duty ratio measured in a robot (normal subject model) experiment are shown in Figure 14. Each circle in those figures means the value of the index measured at the walking cycle. In both stride length and duty ratio, the data of robot experiments (Figure 14) and the data of normal subjects' experiments (Figure 3) show similar 
a

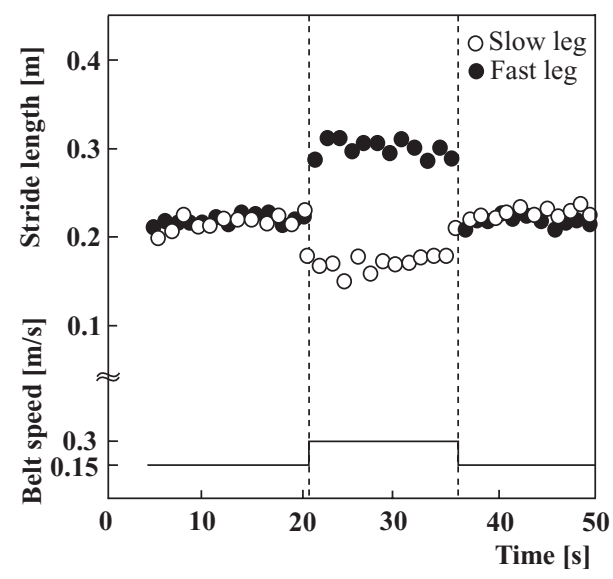

$\mathrm{b}$

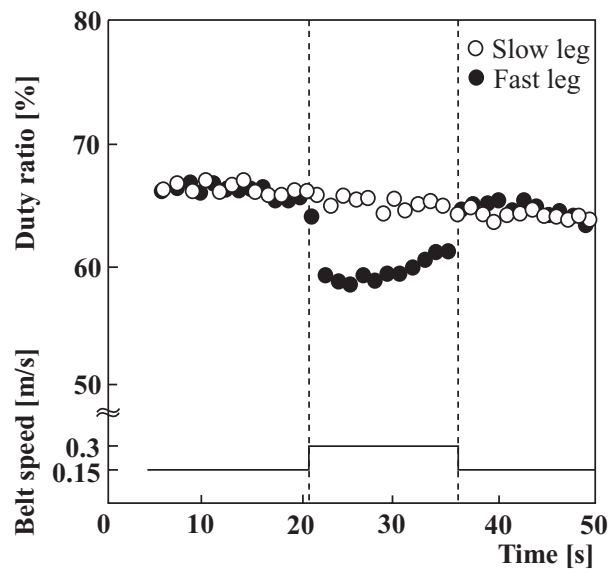

Figure 14. Results of robot (normal subject model) experiment. The stride length:(A) and the duty ratio:(B) in splitbelt treadmill walking are shown, where speed of belts were $0.15 \mathrm{~m} / \mathrm{s}$ at both right and left belts in the baseline stage, $0.15 \mathrm{~m} / \mathrm{s}$ at the right belt and $0.30 \mathrm{~m} / \mathrm{s}$ at the left belt in the adaptation stage, and $0.15 \mathrm{~m} / \mathrm{s}$ at both right and left belts in the post-adaptation stage. Speed of the fast belt is also shown.

values except for the stride length ${ }^{9}$ and similar patterns as described in Section 2.2.

The step length difference and the RDLSP difference measured in a robot (normal subject model) experiment are shown in Figure 15. In both the step length difference and the RDLSP difference, the data of robot experiments (Figure 15) and the data of normal subjects' experiments (Figure 4) show similar values except for the step length difference and similar patterns as described in Section 2.2. The $\psi_{o f f}$ and $k_{h_{-} p}^{s t}$ of both legs in splitbelt treadmill walking are shown in Figure 16. We can see that the hip joint P-gain: $k_{h_{-}}^{s t}$ of the fast leg in the stance phase gradually decreased according to eq. (3) in the adaptation stage. As a result, the decreased $k_{h_{-}}^{s t}$ of the fast leg caused aftereffects on the step length difference and the RDLSP difference at the beginning of the post-adaptation stage as shown in Figure 15.

In summary, Tetsuro (normal subject model) and human (normal subjects) showed similar patterns of those measured indexes in splitbelt treadmill walking.

\subsection{Cerebellar disease subject model}

In order to see the effectiveness of P-gain adjustment and construct the control model of cerebellar disease subjects splitbelt walking, we made Tetsuro walk on the splitbelt treadmill only using joint PD control, cyclic motion trajectory planning and stepping reflex while not using P-gain adjustment. Although Tetsuro without P-gain adjustment (cerebellar disease subject model) could walk on the splitbelt treadmill, the success ratio was lower than that of Tetsuro (normal subject model). In addition, the body position

\footnotetext{
${ }^{9}$ The differences of the stride length and the step length between human and robot experiments are due to the difference of the leg length between human and Tetsuro.
}

in the world coordinate moved forward and backward much more in Tetsuro (cerebellar disease subject model).

In both stride length and duty ratio, the data of robot experiments (Figure 17) and the data of cerebellar disease subjects' experiments (Figure 5) show similar patterns as described in Section 2.3. In both the step length difference and the RDLSP difference, the data of robot experiment (Figure 18) and the data of cerebellar disease subjects' experiments (Figure 6) show similar patterns as described in Section 2.3. Especially, we can see neither gradually returning to the original values in the late adaptation stage, nor aftereffect at the beginning of the post-adaptation stage in Figure 18.

In summary, Tetsuro (cerebellar disease subject model) and human (cerebellar disease subject) showed similar patterns of those measured indexes in splitbelt treadmill walking.

\subsection{Discussions}

\subsubsection{Gait adaptation model}

Reisman et al. (2005) and Morton and Bastian (2006) mentioned that several neural structures including the spinal cord, brainstem, cerebellum and motor cortex have been implicated in the control of various types of locomotor adaptations. However, they also mentioned that it is not exactly known which structures contribute to which types of adaptation through which kind of adjustment mechanisms. Therefore, we proposed adjustment mechanisms for bipedal splitbelt walking, constructed a gait adaptation model of normal subjects and cerebellar disease subjects, and confirmed the validity of the model using a 2D biped robot. Since the data of robot experiments and the data of human experiments showed much similar patterns, we would 


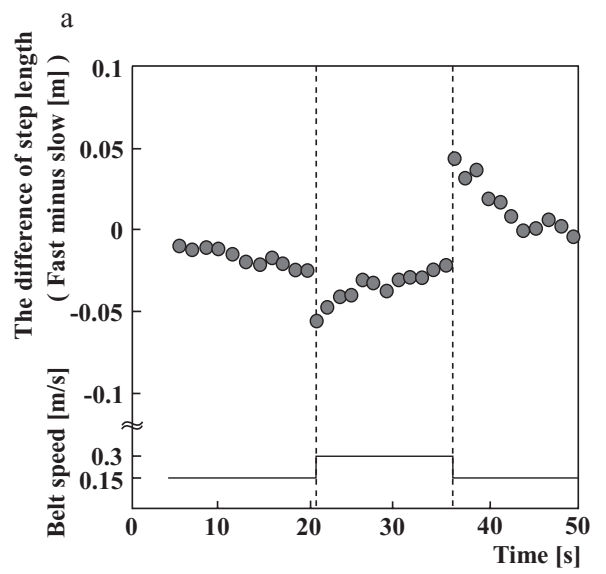

b

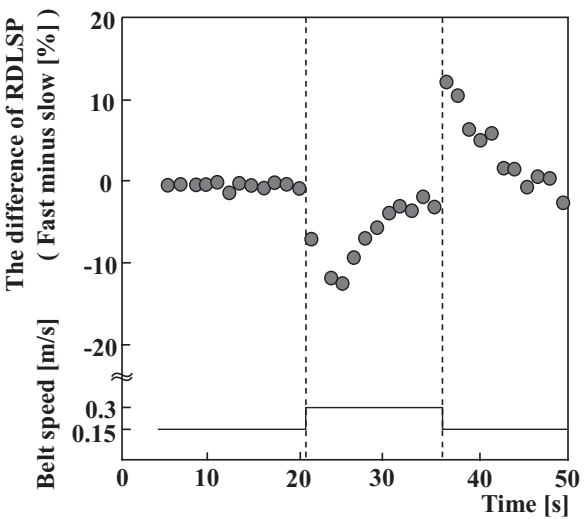

Figure 15. Results of robot (normal subject model) experiment. The step length difference (A) and RDLSP (ratio of the double legs stance period) difference (B) in splitbelt treadmill walking are shown. The time course of belts speed is described in the caption of Figure 14 .
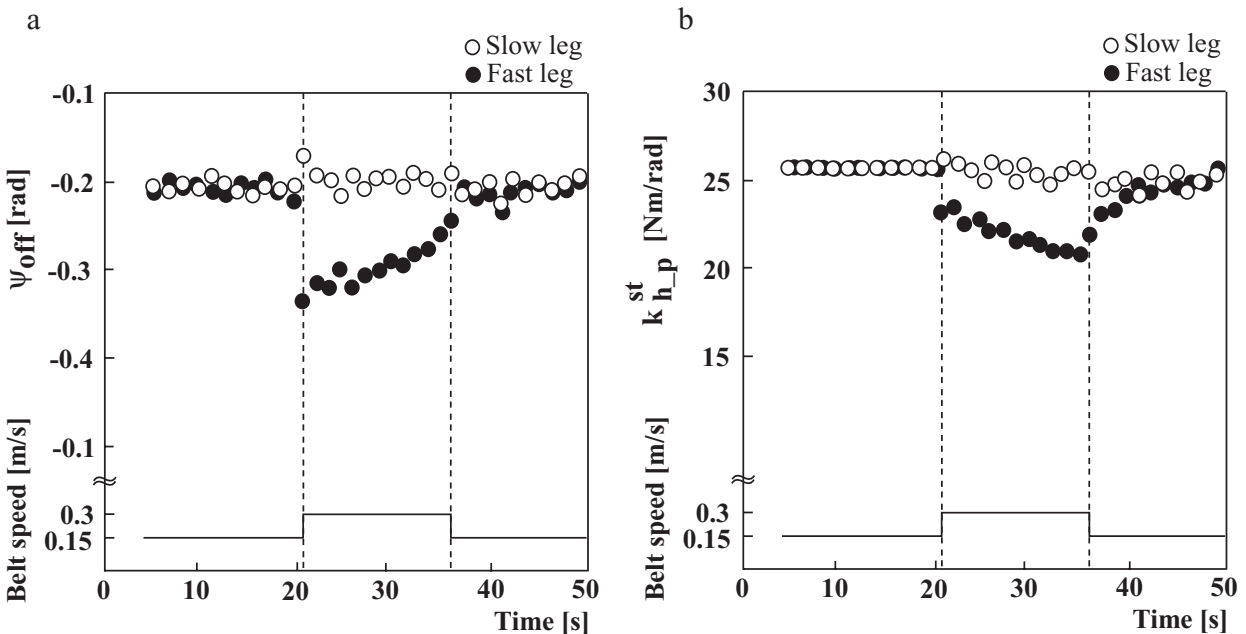

Figure 16. Results of robot (normal subject model) experiment. The $\psi_{o f f}:(\mathrm{A})$ and the hip joint p-gain in the stance phase $k_{h_{-}}^{s t}:(\mathrm{B})$ of both fast and slow legs in splitbelt treadmill walking are shown. The time course of belts speed is described in the caption of Figure 14 .
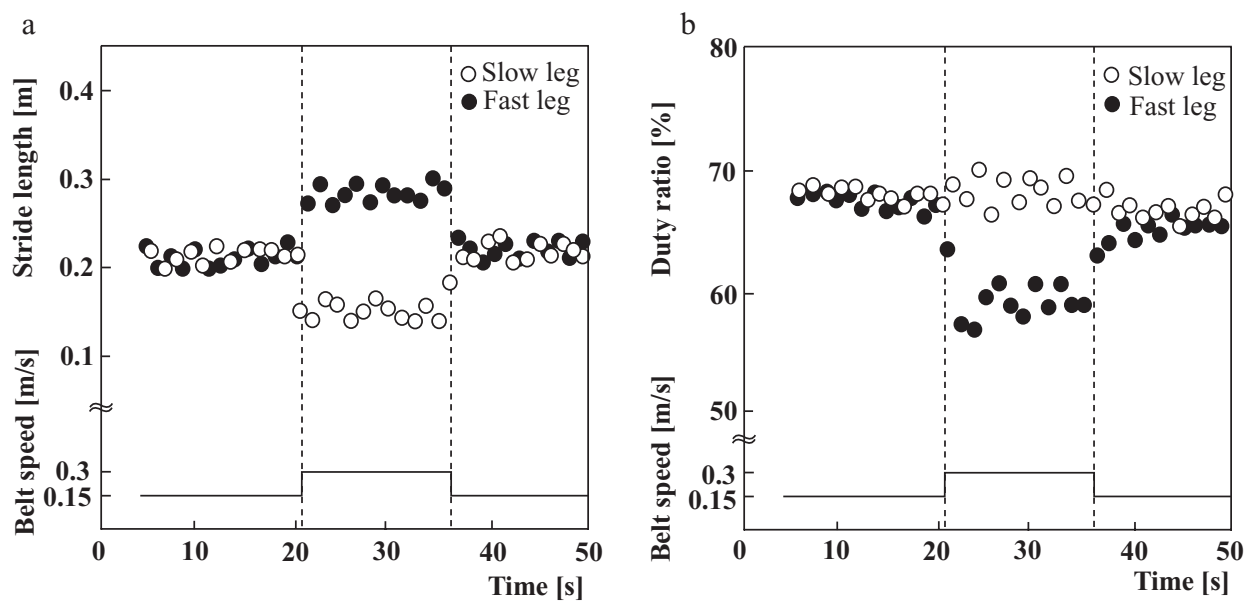

Figure 17. Results of robot (cerebellar disease subject model) experiment. The stride length:(A) and the duty ratio:(B) in splitbelt treadmill walking are shown. The time course of belts speed is described in the caption of Figure 14. 


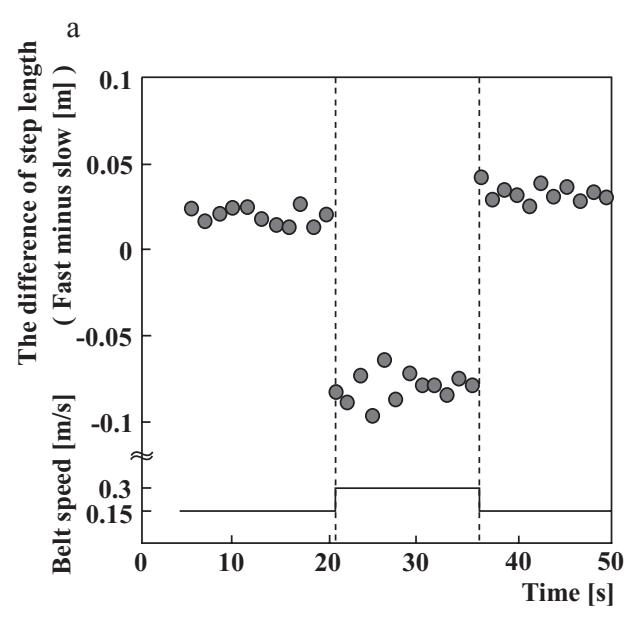

$\mathrm{b}$

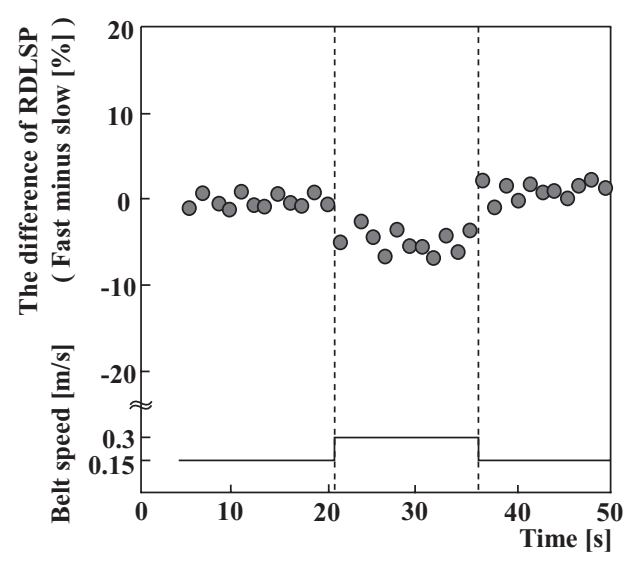

Figure 18. Results of robot (cerebellar disease subject model) experiment. The step length difference (A) and RDLSP difference (B) in splitbelt treadmill walking are shown. The time course of belts speed is described in the caption of Figure 14.

like to suggest that our model approximated human neural structures for bipedal walking to some extent.

As described in Section 2.4 (Reisman et al. 2005; Morton and Bastian 2006), implicitly suggested straightforward adjustment mechanism such that:

(a) The reactive feedback-driven adaptation at the spinal cord or brainstem corresponds to intra-limb coordination adjusting the stride length and the duty ratio.

(b) The predictive feedforward adaptation at the cerebellum corresponds to inter-limb coordination adjusting the step length difference and the RDLSP difference.

But adaptation mechanism in our model (Figure 7) was different from such straightforward adjustment mechanism and summarised as following:

(a) The duty ratio is adjusted mostly passively by kinematical constraints ${ }^{10}$.

(b) The stepping reflex adjusts the intra-limb index (the stride length) in spite of its style as inter-limb control, and corresponds to the reactive feedback-driven adaptation at the brainstem.

(c) P-gain adjustment adjusts inter-limb indexes (the step length difference and the RDLSP difference) in spite of its style as intra-limb control while being combined with the stepping reflex (inter-limb control), and corresponds to the reactive feedback-driven adaptation ${ }^{11}$ at the cerebellum.

\footnotetext{
${ }^{10}$ If we employ a CPG of which phase is reset by PEP (post-exterior position) information of the stance leg (Aoi and Tsuchiya 2005; Morimoto et al. 2008), the duty ratio can be adjusted reactively as being suggested by Reisman et al. (2005) and Morton and Bastian (2006)

${ }^{11}$ In the sense that P-gain adjustment needs $\bar{\psi}_{\text {off }}$ calculated in the baseline stage, it could be goal-directed (Ilg et al. 2007).
}

High similarities in ratios and patterns of the measured indexes between human experiments and robot experiments imply the validity of our hypotheses and the proposed model.

We showed that P-gain at hip joint of the stance leg was the control parameter of adaptation for symmetric gaits in splitbelt walking. But it should be noted that $k_{a g}$ in eq. (3) was determined in relation to $k_{s r}$ in (2) since P-gain adjustment worked well while being combined with the stepping reflex in our model as described in Section 3.3. It is difficult to conclude the P-gain adjustment is more reasonable than more straightforward approaches such as the phase reset (Aoi and Tsuchiya 2005; Morimoto et al. 2008) or the referential ZMP relocation (Hirai et al. 1998). There is no other research to construct the gait adaptation model on the splitbelt treadmill as we have known. We need to carry out experiments using these approaches to make sure whether P-gain adjustment is really reasonable or not. P-gain adjustment first works combined with the stepping reflex on the splitbelt treadmill. The stepping reflex is similar to the phase reset (Aoi and Tsuchiya 2005; Morimoto et al. 2008) from the view of limit-cycle. Both the stepping reflex and phase reset are limit-cycle patterns achieved by adaptive walking. We have just picked up the one pattern from them. So, a gait adaptation model using the phase reset does have a chance to show the similar results from P-gain adjustment. Tetsuro both with P-gain adjustment (normal subject subject model) and without P-gain adjustment (cerebellar disease subject model) could walk on the splitbelt treadmill. Consequently, the success ratio of the cerebellar disease subject model was lower than that of the normal subject model. The success ratio of the normal subject model was $65 \%$ (65 time success of 100 trials) and that of the cerebellar disease subject model was $45 \%$ (45 time success of 100 trials). It means the proposed proportional gain adjustment stabilises splitbelt treadmill walking. 


\subsubsection{Future works}

As the next step, we would like to expand the proposed control model using a biped robot which has a torso and can walk in 3D without constraint in the roll plane. But in order to investigate why and how our control model adjusts those indexes in robot splitbelt walking, we have to carry out mathematical analysis. Specifically, we want to construct the dynamic model of Tetsuro, declare the variables of joint angles, walking cycle, belt speed and so on and represent the four kinematic indexes, i.e. stride length, step length, duty ratio and RDLSP, in the form of equations. In addition, in order to confirm the validity of our hypotheses and the proposed model, we have to arrange another experiment of human splitbelt walking in future. We consider that the proposed controller works well to adapt whether the robot has a torso or not, because it is constructed referring to the knowledge in physiology and hypotheses by Reisman et al. (2005) and Morton and Bastian (2006). But we need the additional posture control to stabilise the torso when the robot has one.

\section{Conclusion}

In order to construct the gait adaptation model of human splitbelt treadmill walking, we proposed a simple control model and made a newly developed 2D biped robot walk on the splitbelt treadmill. We combined the conventional limit-cycle based control consisting of joint PD-control, cyclic motion trajectory planning and the stepping reflex with a newly proposed adjustment of P-gain at the hip joint of the stance leg. We showed that the data of robot (normal subject model and cerebellum disease subject model) experiments had high similarities with the data of normal subjects' and cerebellum disease subjects' experiments carried out by Reisman et al. (2005) and Morton and Bastian (2006) in ratios and patterns and got close to the data of normal subject and cerebellar disease subject in kinematics by using the P-gain adjustment or not. Consequently, we successfully proposed the gait adaptation model in human splitbelt treadmill walking and confirmed the validity of our hypotheses and the proposed model using the biped robot.

\section{Acknowledgements}

This work has been partially supported by a Grant-in-Aid for Scientific Research on Priority Areas 'Emergence of Adaptive Motor Function through Interaction among Body, Brain and Environment' from the Japanese Ministry of Education, Culture, Sports, Science and Technology.

\section{References}

Aoi S, Tsuchiya K. 2005. Locomotion control of a biped robot using nonlinear oscillators. Auton Robots. 19:219-232.

Asano F, Luo Z, Yamakita M. 2004. Some extensions of passive walking formula to active biped robots. Proceedings of IEEE ICRA, New Orleans, 3797-3802.
Chemori A, Alamir M. 2004. Generation of multi-steps limit cycles for rabbit using a low dimensional nonlinear predictive control scheme. Proceedings of IEEE/RSJ IROS, Sendai, 3:2259-2264.

Collins SH, Wisse M, Ruina A. 2001. A three-dimensional passive-dynamic walking robot with two legs and knees. Int J Robotics Res. 20:607-615.

Endo G, Morimoto J, Nakanishi J, Cheng G. 2004. An Empirical exploration of a neural oscillator for biped locomotion control. Proceedings of IEEE ICRA, New Orleans, 3036-3042.

Geng T, Porr B, Wörgötter F. 2006. Fast biped walking with a sensor-driven neuronal controller and real-time online learning. Int J Robot Res. 25:243-259.

Hirai K, Hirose M, Haikawa Y, Takenaka T. 1998. The development of Honda Humanoid Robot. Proceedings of IEEE ICRA, Leuven, p. 1321-1326.

Ijspeert AJ. 2008. Central pattern generators for locomotion control in animals and robots: a review. Neural netw. 21:642-653.

Ijspeert AJ, Crespi A, Ryczko D, Cabelguen J. 2007. From swimming to walking with a salamander robot driven by a spinal cord model. Science. 9:1416-1420.

Ilg W, Golla H, Thier P, Giese A. 2007. Specific influences of cerebellar dysfunctions on gait. Brain. 130:786-798.

Kajita S, Tani K. 1997. Adaptive gait control of a biped robot based on realtime sensing of the ground profile. Auton Robots. 4:297-305.

Kandel ER, Schwartz JH, Jessell TM. 1996. Principles of neural science. Norwalk (CT): Appleton \& Lange, p. 626-646.

Maufroy C, Kimura H, Takase K. 2008. Towards a general neural controller for quadrupedal locomotion. Neural netw. 21:667681.

Miura H, Shimoyama I. 1984. Dynamical walk of biped locomotion. Int J Robotics Res. 3:60-74.

Morimoto J, Endo G, Nakanishi J, Cheng G. 2008. A biologically inspired biped locomotion strategy for humanoid robots: modulation of sinusoidal patterns by a coupled oscillator model. IEEE Trans Rob. 24:185-191.

Morton SM, Bastian AJ. 2006. Cerebellar contributions to locomotor adaptations during splitbelt treadmill walking. J Neurosci. 26:9107-9116.

Nishiwaki K, Kagami S. 2008. Short cycle pattern generation for online walking control system of humanoids. Berlin: Springer Tracts Adv Robotics, 39:541-550.

Orlovsky G, Deliagina T, Grillner S. 1999. Neural control of locomotion. New York: Oxford University Press.

Otoda Y, Kimura H, Takase K. 2007. Efficient bipedal walking using delayed stepping seflex. Proceedings of SICE Annual Conference 2007 (SICE2007), Takamatsu, p. 2170-2174.

Raibert MH. 1986. Legged robots that balance. Cambridge (MA): The MIT Press.

Reisman DS, Block HJ, Bastian AJ. 2005. Interlimb coordination during locomotion: what can be adapted and stored?. J Neurophysiol. 94:2403-2415.

Rogers MW, Johnson ME, Martinez KM, Mille M, Hedman LD. 2003. Step training improves the speed of voluntary step initiation in aging. J Gerontol Ser A: Biol Sci Med Sci. 58:4651.

Rossignol S, Dubuc R, Gossard J. 2006. Dynamic sensorimotor interactions in locomotion. Physiol Rev. 86:89-154.

Sano A, Furusho J. 1990. Realization of natural dynamic walking using the angular momentum information. Proceedings of IEEE ICRA, Cincinnati, 3:1476-1481.

Schaal S, Nakamura Y, Dario P. 2008. Special issue on robotics and neuroscience. Neural networks, 21:551-52.

Sugimoto Y, Osuka K. 2002. Walking control of quasi-passivedynamic-walking robot Quartet III based on delayed feedback 
control. Proceedings of the 5th International Conference on Climbing and Walking Robots, Paris, p. 123-130.

Taga G, Yamaguchi Y, Shimizu H. 1991. Self-organized control of bipedal locomotion by neural oscillators. Biolog Cybern. 65:147-159.

Takanishi A, Ishida M, Yamazaki Y, Kato I. 1985. The realization of dynamic walking by the biped walking robot WL-10RD, Proceedings of IEEE ICAR, Tokyo, p. 459-466.

Takuma T, Hosoda K. 2006. Controlling the walking period of a pneumatic muscle walker. Int J Robotics Res. 25:861-866.

Tomita N, Yano M. 2003. A model of learning free bipedal walking in indefinite environment - constraints self-emergence/selfsatisfaction paradigm. Proceedings of SICE Annual Conference, Fukui, p. 3176-3181.

Tsujita K, Inoura T, Morioka A, Nakatani K, Suzuki K, Masuda T. 2007. Oscillator-controlled bipedal walk with pneumatic actuators. J Mech Sci Technol. 21:976-980.

Wisse M, van Frankenhuyzen J. 2006. Design and construction of MIKE: a 2D autonomous biped based on passive dynamic walking. In: Adaptive motion of animals and machines, Tokyo: Springer, p. 141-152.

Yamaguchi J, Takanishi A. 1997. Development of a leg part of a humanoid robot-development of a biped walking robot adapting to the humans' normal living floor. Auton Robots. 4:369-385.

Yokoi K, Kanehiro F, Kaneko K, Kajita S, Fujiwara K, Hirukawa H. 2004. Experimental study of humanoid robot HRP-1S. Int J Robotics Res. 23:351-362.

\section{Appendix}

\section{A. Trajectory planning and basic experiment}

\section{A.1 Trajectory planning}

In Figure 9, each joint angle is measured as a relative angle, rather than an absolute angle. The rotation to the flexor direction is positive for each joint. In this study, first three steps from stationarily standing are called 'transitional walking' and afterward steps are called 'steady walking' even in splitbelt treadmill walking.

A single step is programmatically separated into two states, those are, 'stance-swing state' and 'landing-exchange state', and the periods of two states are set to $T_{0}$ and $\Delta T$, respectively. As a result, the period of a single step is $T_{0}+\Delta T$.

\section{Trajectory based on inverted pendulum model}

A 2D inverted pendulum model shown in Figure 19 is used in order to plan the trajectory of the stance leg in the transitional walking and to determine the touchdown angle of the swing leg in whole walking. The equation of motion and constraints ${ }^{12}$ to obtain the motion of the inverted pendulum are expressed as follows.

$$
\begin{gathered}
I_{1} \ddot{\phi}=m_{1} g l_{1} \phi, \\
\phi(0)=-\phi\left(T_{0}\right), \\
\dot{\phi}(0)=\dot{\phi}\left(T_{0}\right),
\end{gathered}
$$

where $I_{1}, m_{1}, l_{1}$ and $g$ are moment of inertia around the joint, mass of link1, the length of link1 and acceleration of gravity, respectively. The trajectory that satisfies these three equations in

\footnotetext{
${ }^{12}$ We assume the inelastic collision at foot contact and the law of conservation of angular momentum.
}

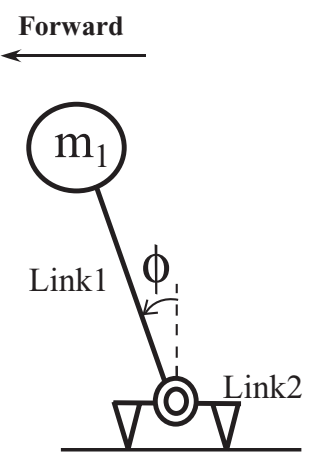

Figure 19. Inverted pendulum as a simplified model of Tetsuro. Although the sole of the stance leg rotates with respect to the ground in Tetsuro (Figure 8-(d)), we ignore such effect in this model.

a single step $\left(0 \leq t \leq T_{0}\right)$ is expressed as follows:

$$
\phi(t)=\left(1+e^{-a T_{0}}\right) e^{a t}-\left(1+e^{a T_{0}}\right) e^{-a t}, \quad a=\sqrt{m_{1} g l_{1} / I_{1}}
$$

\section{Stance-swing state}

In the stance-swing state, the trajectories for the single leg stance phase are planned. In this period, $0 \leq t \leq T_{0}$. The desired trajectories in the stance-swing state are shown in Table 3. In the swing leg, the desired angle of the hip joint is set using eq. (7). The knee joint is free to utilise natural dynamics. The desired angle of the ankle joint is set to raise its toe a little. In the stance leg, the desired angle of the hip joint is set using eq. (7). The knee joint is locked by its motor and the mechanical stopper (Figure 8(c)). The desired angle of the ankle joint is constant in steady walking. As a result, the stance leg becomes a single link inverted pendulum with virtually passive spring-dumper. When $t<T_{0}$, the planned motion enters the landing-exchange state.

\section{Landing-exchange state}

In the landing-exchange state, the swing leg lands on ground, and the swing leg and the stance leg are exchanged in the double legs stance period (Figure 20). The desired trajectories in the landing-exchange state is shown in Table 2. During this period,

Table 2. Desired trajectory of each joint in the landing-exchange state.

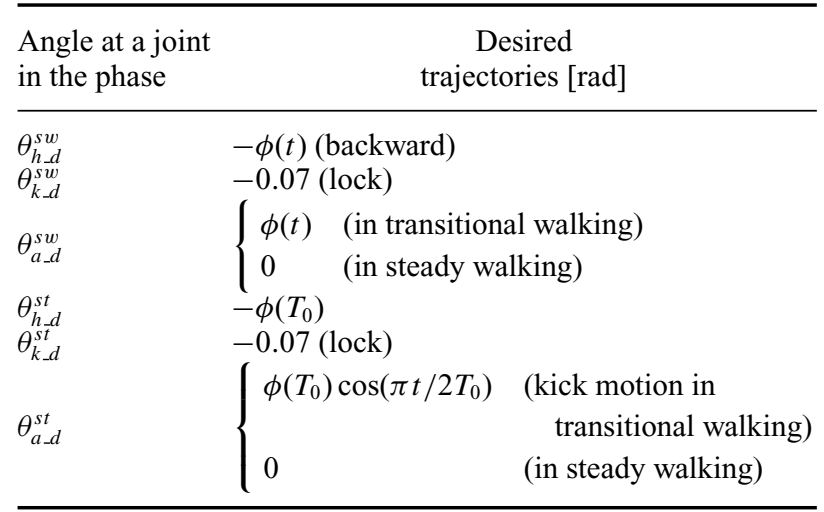




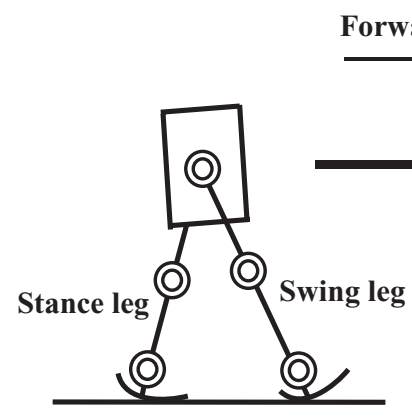

(a)

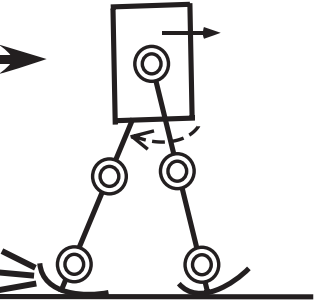

(b)
Figure 20. When the swing leg touches the ground (a), the double legs stance period appears. Here, we still call the forward leg as 'swing leg' and the backward leg as 'stance leg'. The knee joints of swing and stance legs are mechanically locked. In addition, the desired trajectory as kick motion is given to the ankle joint of the stance leg (b) to help the body move forward only in the transitional walking.

$T_{0}<t \leq T_{0}+\Delta T$. Backward motion of the swing leg is planned at the hip joint in order for the swing leg to land on ground and move the body forward after landing (Figure 20). The desired trajectory of kick motion is given to the ankle joint of the stance leg to help the body move forward only in the transitional walking (Figure 20). This trajectory was obtained by trial and error. The knee joints of both legs are mechanically locked. The time $t$ is reset to zero at $t=T_{0}+\Delta T$, and stance and swing legs are exchanged and the planned motion moves to the next step.

\section{A.2 Overview of planned motion and real motion}

The time sequences of planned motion and real motion are shown in Figure 21. We plan the motion of Tetsuro as the time sequences in a top-down style as described in Appendix A.1. But the real motion of Tetsuro is generated as the result of interaction among the controller, mechanisms and ground. Therefore, the duty ratio of a leg is variable according to the speed of the belt in splitbelt treadmill walking (Figure 21). The leg phase (stance or swing) is measured by contact sensors. As a result, whether Tetsuro is in the single leg stance period (SLSP) or the double legs stance period

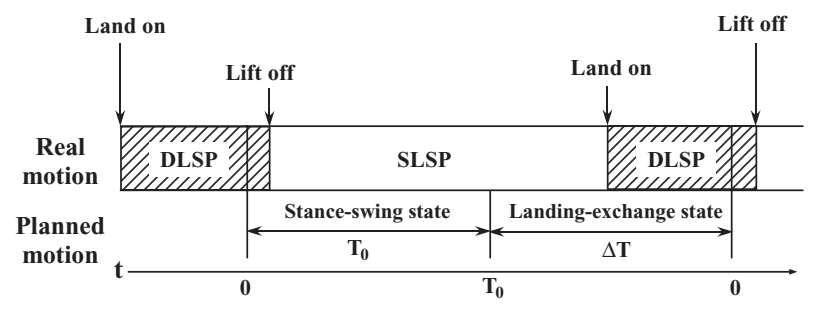

Figure 21. Overview of planned motion and real motion. SLSP and DLSP mean the single leg and double legs stance period, respectively. The timings of landing on and lifting off ground depend on the relation between the real motion of Tetsuro and ground. In this figure, the SLSP starts a little delayed from the start of the stance-swing state, and ends much delayed from the end of the stance-swing state. The time $t$ in Table 3.2.5 and Table 5 is reset to zero at $t=T_{0}+\Delta T$, and stance and swing legs are exchanged.

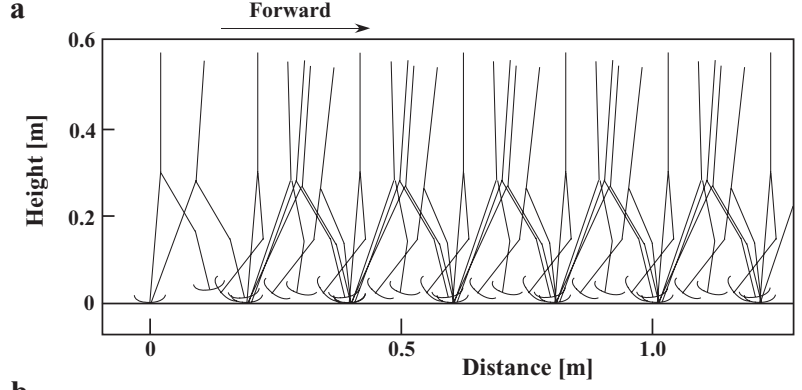

b

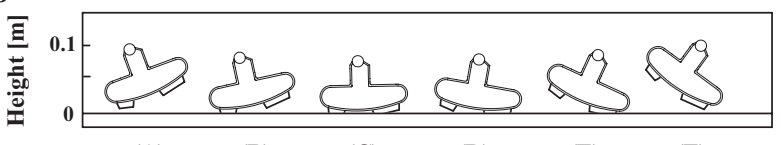

$\begin{array}{llllll}\text { (A) } & \text { (B) } & \text { (C) } & \text { (D) } & \text { (E) } & \text { (F) }\end{array}$

Figure 22. Experimental results of robot tied treadmill walking with the belt speed: $0.15 \mathrm{~m} / \mathrm{s}$. The stick diagram (A), and motion of a foot (B) in landing (a), stance (b) (e) and liftoff (f) are shown. The distance was calculated using measured joint angles and the body pitch angle. Of course, Tetsuro moved forward or backward a little on the treadmill, and belts moved to backward.

(DLSP) is determined. DLSP appears twice in single walking cycle as described in Section 2.1.

\section{A.3 Experimental results of tied treadmill walking}

Tetsuro successfully walked on the tied configuration treadmill with the belt speed: $0.15 \mathrm{~m} / \mathrm{s}$ using above described simple PD control and planned trajectory at each joint while setting $T_{0}=0.32$ and $\Delta T=T_{0}$. Tetsuro was standing stationarily at beginning, and automatically started walking when the treadmill started moving.

In Figure 22(a), the stick diagram is drawn using measured joint angles while considering the body pitch angle calculated by a rate gyro sensor output. We can see stable walking on the treadmill. The stride length was approximately $0.2 \mathrm{~m}$. In Figure 22(b), motion of a foot is shown. We can see 'heel strike and toe off' motion like human usual walking. The actual walking cyclic period, the duration of single DLSP (double legs stance period) and the duty ratio were approximately $1.3 \mathrm{~s}, 0.18 \mathrm{~s}$ and 0.72 . In Figure 23, snapshots of Tetsuro in tied treadmill walking are shown. We can

Table 3. Values of the parameters used in experiments with Tetsuro. SSS and LES mean the stance-swing state and landingexchange state, respectively.

\begin{tabular}{|c|c|c|c|}
\hline Parameters & Value & Parameters & Value \\
\hline$I_{1}\left[\mathrm{~kg} \cdot \mathrm{m}^{2}\right]$ & 0.39 & $k_{k_{-} p}^{s t}[\mathrm{Nm} / \mathrm{rad}]($ in SSS) & 9.0 \\
\hline$m_{1}[\mathrm{~kg}]$ & 2.3 & $k_{a_{-} p}^{s t}[\mathrm{Nm} / \mathrm{rad}]($ in SSS) & 3.0 \\
\hline$g\left[\mathrm{~m} / \mathrm{s}^{2}\right]$ & 9.8 & $k_{h_{-} p}^{s t}[\mathrm{Nm} / \mathrm{rad}]$ (in LES) & 18 \\
\hline$l_{1}[\mathrm{~m}]$ & 0.41 & $k_{k_{-} p}^{s t}[\mathrm{Nm} / \mathrm{rad}]$ (in LES) & 9.0 \\
\hline$T_{0}[\mathrm{~s}]$ & 0.32 & $k_{a_{-} p}^{s t}[\mathrm{Nm} / \mathrm{rad}]$ (in LES) & 2.0 \\
\hline$k_{h_{-} p}^{s w}[\mathrm{Nm} / \mathrm{rad}]$ (in SSS) & 26 & $k_{h_{-} p}^{s w}[\mathrm{Nm} / \mathrm{rad}]$ (in LES) & 20 \\
\hline$k_{k_{-}}^{s w}[\mathrm{Nm} / \mathrm{rad}]($ in SSS) & 0.0 & $k_{k_{-} p}^{s w}[\mathrm{Nm} / \mathrm{rad}]$ (in LES) & 9.0 \\
\hline$k_{k_{-} v}^{s w}[\mathrm{Nm} \cdot \mathrm{s} / \mathrm{rad}]$ (in SSS) & 0.0 & $k_{a_{-} p}^{s w}[\mathrm{Nm} / \mathrm{rad}]($ in LES) & 3.0 \\
\hline$k_{a_{-} p}^{s w}[\mathrm{Nm} / \mathrm{rad}]($ in SSS) & 2.0 & $k_{s r}[\mathrm{~s}]$ & 0.35 \\
\hline$k_{h_{-}}^{s t}[\mathrm{Nm} / \mathrm{rad}]$ (in SSS) & 26 & $k_{a g}\left[\mathrm{Nm} / \mathrm{rad}^{2}\right]$ & 3.9 \\
\hline
\end{tabular}




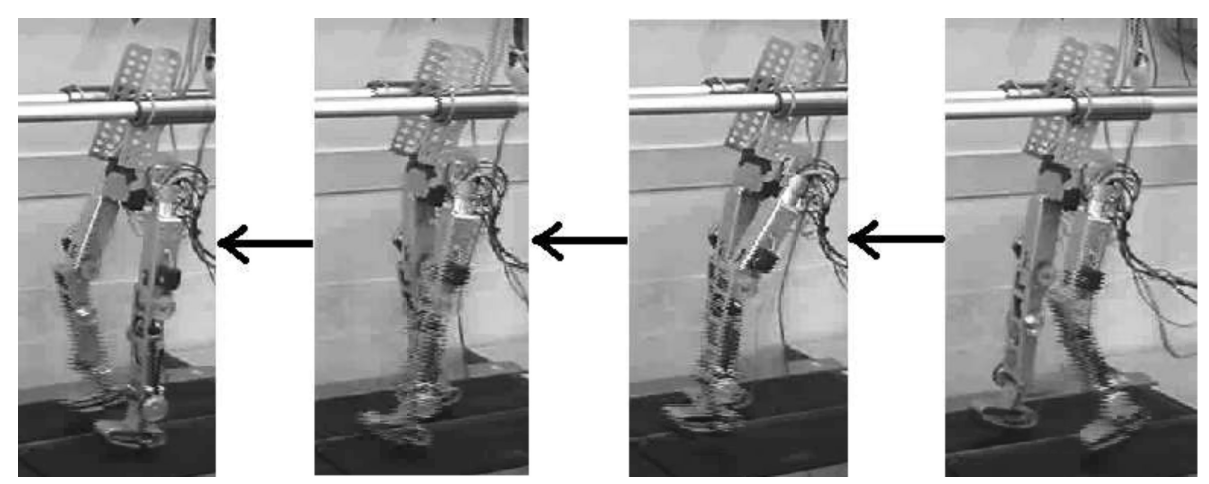

Figure 23. Snapshots on tied treadmill walking with the belt speed: $0.15 \mathrm{~m} / \mathrm{s}$.

see that the knee joint of the stance leg is not bending as usual human walking.

\section{B. Values of parameters}

\section{Additional experiments}

We carried out experiments in the other splitbelt configuration. The speed of the treadmill belt was either 'slow' $(0.15 \mathrm{~m} / \mathrm{s})$ or 'slower' $(0.075 \mathrm{~m} / \mathrm{s})$. The time course of robot experiments was same with the one shown in Figure 1. The period of each stage was $15 \mathrm{~s}$. We also made Tetsuro walk on the splitbelt treadmill in the above described experimental setup with the P-gain adjustment described in Section 3.3. The $\psi_{\text {off }}$ and $k_{h_{-}}^{s t}$ of both legs in splitbelt treadmill walking are shown in Figure 24. We can see that the hip joint P-gain: $k_{h_{-}}^{s t}$ of the fast leg in the stance phase quickly changed at the beginning of the adaptation stage, gradually decreased in the stage and gradually returned almost to the original values in the post-adaptation stage according to eq. (3). The P-gain adjustment made Tetsuro walk on the splitbelt treadmill which one treadmill belt was set at slower speed in the adaptation stage.
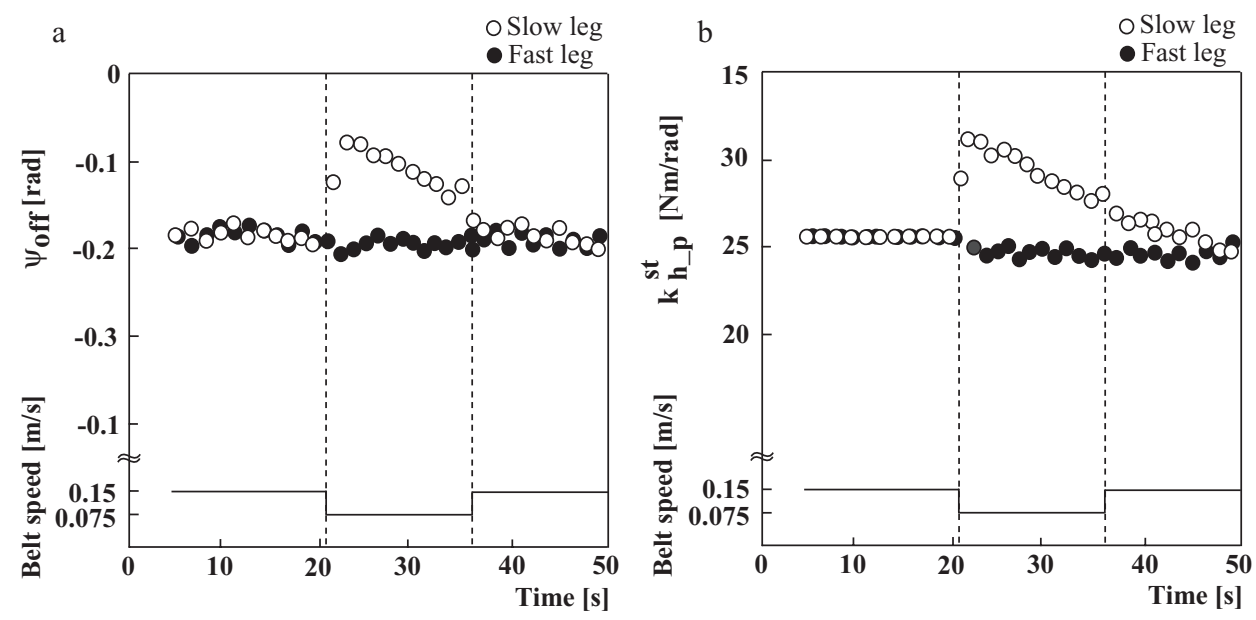

Figure 24. Results of robot (normal subject model) experiment. The $\psi_{\text {off }}:(\mathrm{A})$ and the hip joint p-gain in the stance phase $k_{h-p}^{s t}:(\mathrm{B})$ of both fast and slow legs in splitbelt treadmill walking are shown, where speed of belts were $0.15 \mathrm{~m} / \mathrm{s}$ at both right and left belts in the baseline stage, $0.15 \mathrm{~m} / \mathrm{s}$ at the right belt and $0.075 \mathrm{~m} / \mathrm{s}$ at the left belt in the adaptation stage, and $0.15 \mathrm{~m} / \mathrm{s}$ at both right and left belts in the post-adaptation stage. Speed of the fast belt is also shown. 

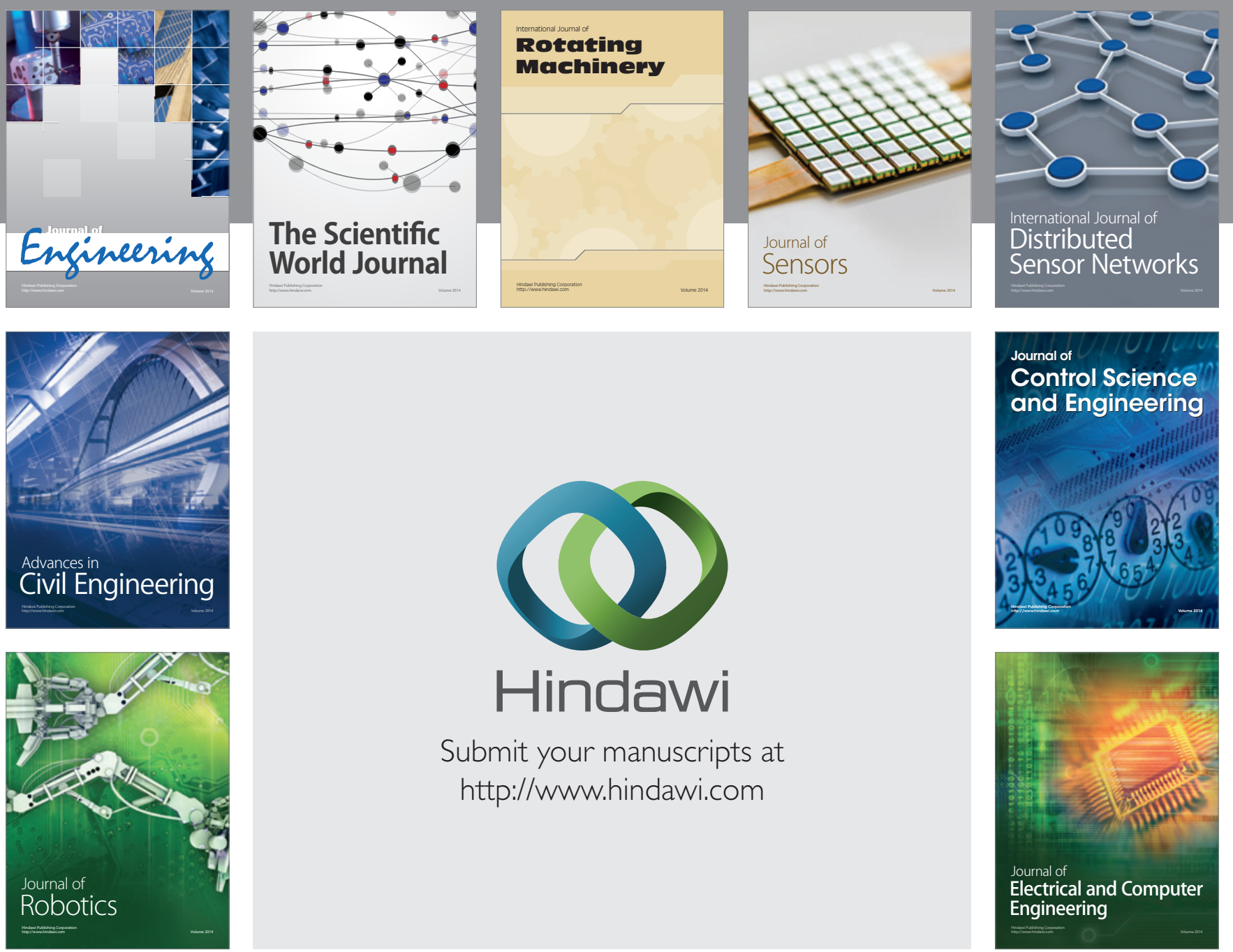

Submit your manuscripts at

http://www.hindawi.com
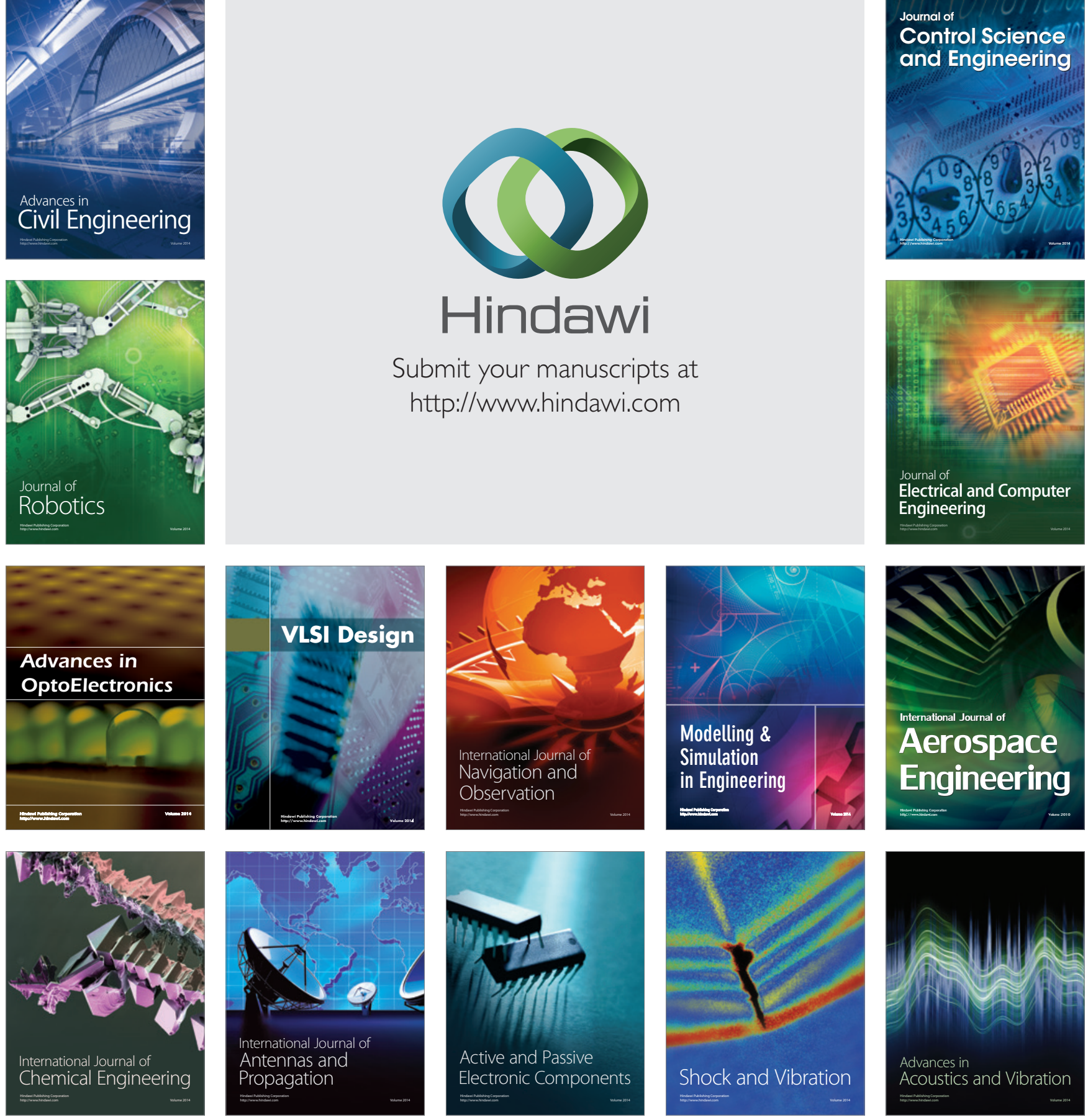